\title{
Type specimens of Pompiloidea, Thynnoidea and Vespoidea (Hymenoptera) deposited in the Museu de Zoologia da Universidade de São Paulo, Brazil
}

\author{
Tamires de 0. Andrade ${ }^{1,2}$; Kelli S. Ramos ${ }^{1,3}$; Helena C. Onody ${ }^{1,4}$; Alvaro D. dos Santos ${ }^{1,5}$ \& Carlos Roberto F. Brandão, \\ 1 Universidade de São Paulo (USP), Museu de Zoologia (MZUSP). São Paulo, SP, Brasil. \\ 2 ORCID: 0000-0001-9492-1967. E-mail: tamiresandrade@usp.br \\ 3 ORCID: 0000-0003-1453-5885.E-mail: kellisramos@gmail.com \\ 4 ORCID: 0000-0003-3570-8183. E-mail: helenaonody@gmail.com \\ ${ }^{5}$ ORCID: 0000-0002-7997-4195. E-mail: alvarods3@gmail.com \\ ${ }^{6}$ ORCID: 0000-0002-4689-5845. E-mail: crfbrand@usp.br
}

\begin{abstract}
The Hymenoptera collection of the Museu de Zoologia da Universidade de São Paulo, Brazil (MZSP) houses one of the most important collections for the Brazilian and Neotropical wasp faunas. The present catalogue lists the type specimens of Pompiloidea, Thynnoidea and Vespoidea (Insecta, Hymenoptera) deposited in the MZSP. The collection includes a total of 422 type specimens ( 66 holotypes, 28 lectotypes, 278 paratypes, 45 paralectotypes, and five syntypes), being 91 Pompiloidea (40 Mutillidae type specimens of 10 genera and 25 species, 51 Pompilidae type specimens of 4 genera and 27 species), 191 Vespoidea (15 genera and 86 species of Vespidae), and 18 Thynnoidea (eight genera and 12 species of Thynnidae). All information written in labels was compiled and is presented along with additional data from the original descriptions, MZSP records and other sources. We provide Specimage links to high-resolution photographs of holotypes and lectotypes at the Hymenoptera image database of the Ohio State University.
\end{abstract}

Key-Words. Catalogue; Insecta; MZSP; Taxonomy; Wasp.

\section{INTRODUCTION}

The collection of Pompiloidea, Thynnoidea and Vespoidea (Hymenoptera) housed in the Museum of Zoology of the University of São Paulo (MZSP) is one of the most important scientific collections for the Brazilian and Neotropical wasp faunas, regarding the number of types, species and specimens, as well as taxonomic and geographic coverage. The earliest records of the collection date back to the early $20^{\text {th }}$ century, when the collection still belonged to the Zoology section of the 'Museu Paulista', with material collected mainly by Hermann von Ihering (1850-1930) and by Hermann von Lüderwaldt (1865-1934) (Klingenberg \& Brandão, 2005). Since then, the collection received several type specimens described by Adolpho Ducke, Clarence E. Mickel, Osvaldo H. Casal, Francisco J. Suárez, William J. Fox, Jean Brèthes, Colin R. Vardy, Lynn S. Kimsey, Hermann Friederich von Ihering, Joseph Zikán, and Owain W. Richards. Addionally, research projects such as the recent programs Biota/FAPESP, INCT-HYMPAR/Sudeste (CNPq/FAPESP), and PROTAX (CNPq/FAPESP) have collaborated greatly for the growth and maintenance of the collection.
This paper is part of a series of lists being published to cover the Hymenoptera type specimens deposited in the entomological collection of MZSP. Until this moment, the following catalogues have been published: ants (Brandão, 1991; Klingenberg \& Brandão, 2005; Brandão et al., 2010; Esteves et al., 2011; Prado \& Brandão, 2013; Ulysséa \& Brandão, 2013; Ulysséa et al., 2015), bees (Ramos et al., 2015), "Symphyta" \& "Parasitica" wasps (Onody et al., 2014) and Chrysidoidea wasps (Santos et al., 2017). These initiatives agree with the $72 \mathrm{~F}$ of the International Code of Zoological Nomenclature (ICZN, fourth edition, January 1999) recommendation making information about these types available to researches. Additionally, images are also given complementing the catalogue and hopefully avoiding unnecessary movement of these unique specimens.

\section{MATERIAL AND METHODS}

In this catalogue, we adopted the classification of Pilgrim et al. (2008), which recognizes eight superfamilies in Aculeata: Apoidea, Chrysidoidea, Formicoidea, Pompiloidea, Scolioidea, Tiphioidea, 
Thynnoidea and Vespoidea. The MZSP collection has no types of Scolioidea and Tiphioidea; Chrysidoidea and Formicoidea types were already catalogued and Apoidea catalogue is in preparation. To address the type specimen's current status, the following references were used: Catálogo Taxonômico da Fauna do Brasil (Oliveira et al., 2018) , Integrated Taxonomic Information System (ITIS), Hymenoptera Online (HOL) and the Checklist and/ or Catalog of Social Wasps (Carpenter, 2018).

All pertinent primary and secondary type specimens deposited at the MZSP were listed according to their original combination and in alphabetical order by family, subfamily, tribe, genus, and species. The names of all species are followed by the name of the author, year and page of the publication. Also, the current valid names are given for each species following by the author's name and year.

For holotypes and lectotypes, a link is provided for high resolution photographs of the specimens in Specimage website (http://specimage.osu.edu) database of The Ohio State University, except the lectotypes of Megacanthopus collaris Ducke, 1904, Mischocyttarus parallelogrammus Zikán, 1935 and Mischocyttarus cristatus Zikán, 1935 that are currently under loan and could not be assessed for this work. For each specimens photographs of labels, head (frontal view), dorsal and lateral habitus are provided. Images were obtained in a Leica M205C magnifying stereoscope attached to a Leica DFC 295 video camera and with a DSLR camera model Cannon 70D and $18-55 \mathrm{~mm}$ lens. Ilumination system employed the low budget dome as proposed by Kawada \& Buffington (2016). We used Leica LAS (Leica Application Suite V3.6.0) and Helicon Focus (Version 6.0.18) to assemble the series of images taken at different focus distances by z-stacking method. Small adjustments, such as brightness and contrast, were made using commercial software.

All information given here was taken from label data, MZSP records, published material and other available sources. The data labels are presented in the following sequence: type category, number and sex of specimens, current MZSP registration number, locality (country, state/province, city, and other locality details), date of collecting, trap and collector's name. The labels were transcribed between apostrophes (' '), indicating information from different labels attached to the same specimen. Abbreviated and additional information are presented in brackets ([ ]). Original data is provided in the original languages of the labels, while additional information is provided in English. Question marks are used when it was not possible to decipher the handwriting.

\section{RESULTS}

Exemplars here listed comprises a total of 301 type-specimens, being 91 of Pompiloidea (Mutillidae: 8 holotypes and 32 paratypes representatives of 10 genera and 25 species, Pompilidae: 20 holotypes, five lectotypes, 19 paratypes, and seven paralectotypes representatives of four genera and 27 species), 191 of Vespoidea (Vespidae: 34 holotypes, 23 lectotypes, 94 paratypes,
38 paralectotypes, and four syntypes representatives of 15 genera and 86 species), and 18 of Thynnoidea (Thynnidae: four holotypes, 13 paratypes and one syntype representatives of eight genera and 12 species).

\section{POMPILOIDEA \\ MUTILLIDAE \\ Mutillinae \\ Trogaspidiini \\ Timulla Ashmead}

\section{Timulla intermissa Mickel, 1938: 606}

Paratypes: 19 (\# MZSP 56445) '[Brazil], Paraná, Rio Negro' 'Paratype, Timulla (T.) intermissa, ㅇ, C.E. Mickel'; 1 ㅇ (\# MZSP 56446) '[Brazil], S.P. [São Paulo], [Cubatão city], Estação Raiz da Serra, 22.xi.1906, João Lima col.' '6.401 [Brazil, São Paulo, Estação Raiz da Serra, 22.xi.1906, João Lima col.]'Paratype, Timulla (T.) intermissa, \&, C.E. Mickel'; 1 (\# MZSP 56447) 'Brasil, SP [São Paulo], São Paulo [city], Ipiranga [neighborhood], 16.iii.1906, H. Luederwaldt col.' '2.543 [Ipiranga, H. Lüderwaldt col., 16.iii.1906]'Paratype, Timulla (T.) intermissa, o, C.E. Mickel'.

\section{Timulla lineoloides Mickel, 1938: 624}

Paratype: 19 (\# MZSP 56448) '[Brazil], S.[ão] Paulo, Cantareira, i.1914, E. Garbe col.'18.697 [Brazil, São Paulo, Cantareira, i.1914, E. Garbe col.]' 'Timulla (T.) lineoloides, \&, C.E. Mickel'.

\section{Timulla lule Casal, 1957: 91}

Paratype: 1 ㅇ (\# MZSP 56449) ‘D.A. [Argentina], Tucuman, [San] Pedro de Colalao, (Arnau), i.1949' 'Col. M.A. Fritz' 'Timulla (T.) lule n. sp., \&, Casal, 1957'.

\section{Timulla odice Mickel, 1938: 578}

Paratypes: 2 9 (\# MZSP 56451-56452) '[Brazil], São Paulo, Alto da Serra, 6.28 [vi.1928], R. Spitz col'Paratype, Timulla (T.) odice, \&, C.E. Mickel'; 1 9 (\# MZSP 56450) '[Brazil], S.[ão] P.[aulo], Estação Raiz da Serra [Cubatão City], 14.i.1906, H. Luederwaldt col.'`8.161 [Brazil, São Paulo, Estação Raiz da Serra, 14.i.1906, H. Lüderwaldt col.]'Paratype, Timulla (T.) odice, \&, C.E. Mickel'; 1 9 (\# MZSP 56454) '[Brazil], S.[ão] Paulo, Estação Raiz da Serra [Cubatão City], 3.xii.1907, H. Luederwaldt col.' '7.049 [Brazil, São Paulo, Cubatão, Estação Raiz da Serra, 3.xii.1907, H. Lüderwaldt col.]' 'Paratype, Timulla (T.) odice, ㅇ, C.E. Mickel'; 1 (# MZSP 56453) '[Brazil], Est.[ado], S.[ão] Paulo, Itanhaem, iv.1928, Spitz col.'`Paratype, Timulla (T.) odice, , , C.E. Mickel'.

\section{Timulla spiniclypeata Mickel, 1938: 604}

Paratype: 19 (\# MZSP 56455) '[Brazil], Est.[ado] São Paulo, Franca, x.1910, E. Garbe col.' '15.772 [Brazil, São Paulo, Franca, x.1910, E. Garbe col.]'Paratype, Timulla (T.) spiniclypeata, ?, C.E. Mickel'. 


\section{Sphaeropthalminae \\ Dasymutillini \\ Cephalomutilla Andre}

\section{Cephalomutilla transversa Mickel, 1960: 166}

Paratypes: 19 (\# MZSP 56404) '[Brazil], Goiás, 11.xi.1906, Baer col.' '6.057 [Brazil, Goiás, 11.xi.1906, Baer col.]' 'Paratype, Cephalomutilla transversa, 9, C.E. Mickel'; 19 (\# MZSP 56405) '[Brazil], Mato Grosso [do Sul], [Porto] Murtinho, xi.1929, R. Spitz col."22.023 [Brazil, Mato Grosso do Sul, Porto Murtinho, xi.1929, R. Spitz col.]' ‘Paratype, Cephalomutilla transversa, , C.E. Mickel'.

Comments: In the original publication the collection date of the specimen from Porto Murtinho (\# MZSP $56405)$ is listed as January, 1930. The original publication also reported one female paratype from São Paulo (Brazil) deposited at MZSP, however, this specimen was not found in the collection.

Traumatomutilla pereirai Suárez, 1960: 473

(http://hol.osu.edu/spmlnfo.html?id=MZSP\%2056442)

Holotype: + (\# MZSP 56442) '[Brazil], Goiás, 1935/36, Spitz col.' 'Traumatomutila pereirai n. sp., J. Suárez det. 1960'.

\section{Traumatomutilla pertela Casal, 1969: 282}

Paratypes: 29 (\# MZSP 56443-5644) 'Brasil, Paraíba, Juazeirinho, Soledade, vi.1956, A.G.A. Silva [col.]' 'Traumatomutilla pertela sp. n. V O.H. Casal, 1968'.

\section{Pseudomethocini Atillum Andre}

\section{Atillum dubium Casal, 1963: 86}

Paratype: 10' (\# MZSP 56403) 'R.A.[Argentina], Bs. As. [Buenos Aires], Est.[ación] F. [Felipe] Sola, A. Martinez [col.]' 'Paratipo' 'Paratypus' 'Atillum dubium sp. nov., Casal $1962,0^{\prime \prime}$

\section{Hoplocrates Mickel}

\section{Hoplocrates scutellaris Mickel, 1941: 363}

Paratypes: 10' (\# MZSP 56406) '[Brazil], Santa Catarina, Colonia Hansa [now Corupá city], 1904, H. Rolle col.' '2.736 [Brazil, Santa Catarina, Joinville, Corupá, 1904, H. Rolle col.'’Paratype, Hoplocrates scutellaris, o', C.E. Mickel'; $10^{7}$ (\# MZSP 56407) '[Brazil], São Paulo, Bocaina, R. Spitz col., tf.24.'Paratype, Hoplocrates scutellaris, o', C.E. Mickel'.

Current status: Hoplocrates specularis (Gerstaecker, 1874).

\section{Hoplomutilla Ashmead}

\section{Hoplomutilla bequaerti Mickel, 1939b: 665}

Paratype: 19 (\# MZSP 56408) '[Brazil], Amazonas, Rio Uaupés, 1907, Bosh col.' '10.779 [Brazil, Amazonas, Rio Uaupés, 1907, Dr. Bosh col.]' 'Paratype, Hoplomutilla bequaerti, $\uparrow$, C.E. Mickel'.

Hoplomutilla lenkoi Suárez, 1961: 225

(http://hol.osu.edu/spmlnfo.html?id=MZSP\%2056409)

Holotype: 9 (\# MZSP 56409) 'Brasil, Rondonia, V.[ila] Rondonia, 378 km S de P. [Porto] Velho, 25.i-9.ii.1961, Pereira e A. Machado [col.]' 'Hoplomutilla lenkoi sp. nov., Holotipo, J. Suárez det. 1961'.

\section{Hoplomutilla myops flavimyops Mickel, 1939a: 377}

Paratype: 1ㅇ (\# MZSP 56410) 'Brasil, Goiás, 11.xi.1906, Baer col.' '6.057 [Brazil, Goiás, 11.xi.1906, Baer col.]' 'Paratype, Hoplomutilla myops ssp. flavimyops, \&, C.E. Mickel'.

\section{Hoplomutilla triumphans Mickel, 1939a: 370}

Paratype: 19 (\# MZSP 56412) '[Brazil], Mato Grosso [do Sul], [Porto] Murtinho, xi.1929, R. Spitz col.'22.023 [Brazil, Mato Grosso do Sul, Porto Murtinho, xi.1929, R. Spitz col.]' 'Paratype, Hoplomutilla triumphans, \&, C.E. Mickel'.

Comments: In the original publication the collection date of the specimen from Porto Murtinho (\# MZSP 56412) is listed as January, 1930.

\section{Lophomutilla Mickel}

Lophomutilla lenkoi Suárez, 1962: 204 (http://hol.osu.edu/spmlnfo.html?id=MZSP\%2056413)

Holotype: O (\# MZSP 56413) 'Brasil, S.[ão] P.[aulo], São Paulo, Interlagos, 4.iii.1961, Reichardt \& Werner' 'Lophomutilla lenkoi sp. nov., §, Holotipo, J. Suárez det. 1962'.

Lophomutilla mickeli Suárez, 1962: 200 (http://hol.osu.edu/spmlnfo.html?id=MZSP\%2056414)

Holotype: ㅇ (\# MZSP 56414)‘Brasil, São Paulo, Salesópolis, Est.[ação] Biol.[ógica] Boraceia, vi.1952, L. Travassos - col' 'Lophomutilla mickeli sp. n., $\$$, Holotipo, J. Suarez det. 1962'.

\section{Lophomutilla prionophora jaragua Casal, 1961: 59}

Paratype: 19 (\# MZSP 56415) 'Brasil, S.[anta] Catarina, Corupá, i.1957, A. Maller' 'Coleção Campos Seabra' 'Lophomutilla prionophora jaragua n. ssp., Casal, 1960, Paratypus, Q'. $^{\prime}$ 


\section{Mickelia Suárez}

\section{Mickelia cressoni Suárez, 1966: 478}

(http://hol.osu.edu/spmlnfo.html?id=MZSP\%2056419)

Holotype: + (\# MZSP 56419) 'Brasil, São Paulo, Ribeirão Preto, 21.x.1962, Exp.[edição] Dep.[artamento] Zool.[ogia]' 'Holótipo, Mickelia cressoni n. sp., ㅇ, J. Suárez det. 1965'.

Paratypes: 19 (\# MZSP 56417)‘[Brazil], M.[ato] Grosso [do Sul], Maracaju, 3-[1]937, Shannon Lane"'Paratipo, Mickelia cressoni n. sp., ㅇ, J. Suárez det. 1965'; 19 (\# MZSP 56418) '[Brazil], São Paulo, Bofete, ii.[1]939, col. Dr. Hempel' 'Paratipo, Mickelia cressoni n. sp., , J. Suarez det.1965'.

\section{Pseudomethoca Ashmead}

\section{Pseudomethoca melanocephala brevipunctata Mickel, 1937: 183}

Paratypes: 19 (\# MZSP 56430) '[Brazil], S.[ão] P.[aulo], Alto da Serra [old name for Paranapiacaba city], Parque Cajuru, iii.1910, H. Luederwaldt col.' '18.551 [Brazil, São Paulo, Paranapiacaba, Parque Cajuru, iii.1910, H. Luederwaldt col.]' 'Paratype, Pseudomethoca melanocephala v. brevipunctata, \&, C.E. Mickel'; 1 \% (\# MZSP 56432) 'Brasil, Est.[ado] S.[ão] P.[aulo], Campos do Jordão, 3.ii.1906, H. Luederwaldt col.' '9.540 [Brazil, São Paulo, Campos do Jordão, 3.ii.1906, H. Luederwaldt col.]"Paratype, Pseudomethoca melanocephala v. brevipunctata, \&, C.E. Mickel'; 19 (\# MZSP 56431) '[Brazil], São Paulo, Capital [São Paulo city], i.1920, Domingos col.' 'Paratype, Pseudomethoca melanocephala v. brevipunctata, \$, C.E.Mickel.'; 1 ' (\# MZSP 56433)’[Brazil], São Paulo, Jundiahy [Jundiaí], xii.1900, M. Beron col.' 'Paratype, Pseudomethoca melanocephala v. brevipunctata, ㅇ, C.E. Mickel'.

\section{Sphaeropthalmini Tallium Andre}

\section{Tallium moleque Casal, 1962a: 323}

Paratype: $10^{`}$ (\# MZSP 56435) ‘Brasil, Paraíba, Juazeirinho, Soledade, Julho, 1956, A.G.A. Silva [col.]'Tallium moleque sp. nov. o', Casal, 1962'.

\section{Tallium precarium Suárez, 1960: 464}

(http://hol.osu.edu/spmlnfo.html?id=MZSP\%2056436)

Holotype: ơ (\# MZSP 56436) '[Brazil], Mato Grosso [do Sul], Maracaju, Shannon Lane, 3.937 [Mato Grosso, Fazenda da Faya, 1905, E. Garbe]"'Tallium precarium n. sp., ơ', J. Suarez det. 1960'.

\section{Tallium schusteri Suárez, 1960: 461}

(http://hol.osu.edu/spmlnfo.html?id=MZSP\%2056437)

Holotype: + (\# MZSP 56437) '[Brazil], Mato Grosso [do Sul], Maracaju, Shannon Lane, 3.937 [Mato Grosso,
Fazenda da Faya, 1905, E. Garbe]' Tallium schusteri n. sp., \$, Holótipo, J. Suarez det. 1960'.

\section{Tallium vianai Casal, 1962b: 274}

Paratype: 19 (\# MZSP 56438) 'Argentina, Cordoba, Dep. [Department] de Calamuchita, "El Sauce", ii.1940, Manuel J. Viana [col.]' 'col. M.A. Fritz'Tallium vianai n. sp. , , Paratypus, Casal, 1960'.

\section{Tallium willinki Suárez, 1960: 468}

(http://hol.osu.edu/spmlnfo.html?id=MZSP\%2056439)

Holotype: ơ (\# MZSP 56439) '[Brazil], Goyaz [Goiás], Campinas, [1]937' 'Tallium willinki n. sp., o', Holótipo, J. Suárez det. 1960'.

\section{Traumatomutilla Andre}

\section{Traumatomutilla maula Casal, 1969: 294}

Paratypes: 29 (\# MZSP 56440-56441) 'Brasil, Pará, Mocajuba, Mocajuba, iii-1953, Orlando M. Rago [col.]' 'Traumatomutilla maula sp. nov.,, , O.H. Casal, 1968'.

\section{POMPILIDAE \\ Pepsinae \\ Pepsini \\ Anacyphonix Banks}

\section{Salius (Priocnemis) apicipennis Fox, 1899: 197} (http://hol.osu.edu/spmlnfo.html?id=MZSP\%2056461)

Holotype: ơ (\# MZSP 56461) '[Brazil, São Paulo, São Paulo], Ypiranga [Ipiranga, neighborhood], ơ', 12.489 [Brazil, São Paulo, São Paulo, Ipiranga, J. Lima col.]"Salius (Priocnemis) apicipennis Fox Fishe!, Fox det.' 'Salius (Priocnemis) apicipennis Fox' 'Salius apicipennis Fox 1899 = Entypus brasiliensis (Taschenberg, 1869), A. Roig Alsina det. 1981"Salius apicipennis Fox 1899, Holotypus, A. Roig Alsina exam. 1981' 'R. Wahis dt. 86, Lep., ơ' 'R. Wahis rv. 86, Holotype, Salius apicipennis Fox 1899, o' = Entypus caerulans, Lep., 1845'.

Current status: Anacyphonyx apicipennis (Fox, 1899).

\section{Entypus Dahlbom}

\section{Salius carinatellus Brèthes, 1911: 68}

(http://hol.osu.edu/spmlnfo.html?id=MZSP\%2056456)

Holotype: ơ (\# MZSP 56456) '[Brazil], E.[stado] de São Paulo, ơ', 12.396 [Brazil, São Paulo]' 'Brèthes det., Salius (Priocnemis) carinatellus n. sp.' 'Salius (Priocnemis) carinatellus n. sp., Breth.' 'Salius carinatellus, Brèthes, 1911, o", Holotypus, A. Roig Alsina exam 1981' 'Salius carinatellus, Brèthes, 1911 = Entypus bituberculatus, (Guérin, 1838), A. Roig Alsina det. 1981"R. Wahis dt.86, Entypus bitubercula- 
tus, Guerin' 'R. Wahis rv. 86, Lectotype, Salius carinatellus, Breth. 1911 = Entypus bituberculatus, Guer. 1838'.

Current status: Entypus bituberculatus (Guérin, 1838).

\section{Salius gracilicornis Brèthes, 1911: 66}

(http://hol.osu.edu/spmlnfo.html?id=MZSP\%2056500)

Holotype: ơ' (\# MZSP 56500) '[Brazil], São Paulo, Capital [São Paulo], ơ', 12.395 [Brazil, São Paulo, São Paulo, 7.v.1902, C. Dreher col.]' 'Salius gracilicornis, Brèthes det.' 'Holotypus, Salius gracilicornis, Brèthes 1911, A. Roig Alsina exam. 1981, Salius gracilicornis'.

Current status: Entypus coeruleus (Taschenberg, 1869).

Comments: We did not find the current information about the status of S. gracilicornis. The association adopted here is based to Banks (1946: 475) in wich refers the holotype male of $S$. gracilicornis may be corresponding to the male of E. coeruleus.

\section{Salius limbatus Brèthes, 1911: 69}

(http://hol.osu.edu/spmlnfo.html?id=MZSP\%2056535)

Holotype: 9 (\# MZSP 56535) '[Brazil], Rio Grande de Sul, O'12.397 [Brazil, Rio Grande do Sul, Mabilde col.]' 'Salius limbatus, Brèthes det.' 'Salius limbatus, Brèthes, 1911, ๆ, Holotypus, A. Roig Alsina exam. 1981' 'Salius limbatus, Brèthes 1911 = Entypus taschenbergii (Dalla Torre, 1897), A. Roig Alsina det. 1981' 'Salius limbatus' 'R. Wehis rv. 86, Holotype, Salius limbatus, Breth, 1911, $9=$ Entypus cyaneus taschenbergii, D. Torre, 1897'.

Current status: Entypus taschenbergi (Dalla Torre, 1897).

Salius (Priocnemis) tinctipennis Fox, 1899: 198

(http://hol.osu.edu/spmlnfo.html?id=MZSP\%2056460)

Lectotype: + (\# MZSP 56460) '[Brazil], Rio Grande do Sul, ${ }^{\prime}$ 'Salius (Priocnemis) tinctipennis Fox, Fishe' 'Salius (Priocnemis) tinctipennis Fox"Salius tinctipennis Fox, 1899, , Lectotypus, A. Roig Alsina exam. 1981"Salius tinctipennis Fox, 1899 = Entypus velutinus (Taschenberg, 1869), A. Roig Alsina det. 1981' 'R.Wahis rv. 86, Lectotype, Salius tinctipennis Fox, 1899, $9=$ Entypus caerulans tinctipennis Fox' 'R. Wahis dt. 86, Entypus caerulans tinctipennis Fox'.

Current status: Entypus velutinus (Taschenberg, 1869).

Comments: Lectotype designated by Roig Alsina (1981:333).

\section{Pepsis Fabricius}

\section{Pepsis achterbergi Vardy, 2005: 191}

Paratype: 19 (\# MZSP 56462) 'Brasil, Esto.[Estado] Pará, Óbidos, (Traira) [community], Dirings [col.]' 'Paratype, + , Pepsis achterbergi n. sp., Det. Vardy, 2001'.

\section{Pepsis adonta Vardy, 2005: 245}

Paratype: 1 († (\# MZSP 56463) '[Brazil], Pará, Rio Xingu, Cach.[oeira] do Espelho, 21-28.x.1986, F.C. do Val. Col.' 'Paratype 9 , Pepsis adonta n. sp., Det. Vardy 2001'.

Comments: The paratype listed herein is incorrectly indicated in the original publication as a male specimen. Also, the male specimen from Canoas (Brazil) listed as deposited at MZSP was not located.

Pepsis bahiae Brèthes, 1914: 288

(http://hol.osu.edu/spmlnfo.html?id=MZSP\%2056464)

Lectotype: (\# MZSP 56464) '[Brazil], Bahia, V.[ila] Nova, Garbe [col.], 14., anno: 1908' Pepsis bahiae, Br., \&, Brèthes, det. 17' 'Lectotype, $q$, Pepsis bahiae Brè. = helvolicornis Luc., det. C.R. Vardy, 1991'.

Paralectotype: 19 (\# MZSP 56465) '[Brazil], Bahia, V.[ila] Nova, Garbe [col.], 14., anno: 1908' 'Pepsis bahiae Br., Brèthes det. 17' '98922' 'Paralectotype, $\$$, Pepsis bahiae Brè. = helvolicornis Luc., det. C.R. Vardy, 1991'.

Current status: Pepsis helvolicornis Lucas, 1895.

Comments: Lectotype designated by Vardy (2005: 114).

Pepsis carinata Brèthes, 1914: 311

(http://hol.osu.edu/spmlnfo.html?id=MZSP\%2056494)

Holotype: ơ (\# MZSP 56494) 'Ecuador, Balzapamba, R. Haensch S. [col]' 'Pepsis carinata Brèth., Brèth. Det. 17.' 'Holotype, o', Pepsis carinata Brè. = purpereipes Pack., det. C.R. Vardy, 1990' 'Pepsis purpureipes Packard, o', Det. Vardy, 92'.

Current status: Pepsis purpureipes Packard, 1869.

Pepsis clypeata Brèthes, 1914: 310

(http://hol.osu.edu/spmlnfo.html?id=MZSP\%2056495)

Holotype: $q$ (\# MZSP 56495) 'Ecuador, Archidona, R. Haensch S. [col]' 'Holotype"'Pepsis clypeata Br., Breth. Det. 17 ' Holotype, +, Pepsis clypeata Brè. = purpurea Sm., det. C.R. Vardy, 1990'.

Current status: Pepsis purpurea Smith, 1873.

Pepsis coronaria Brèthes, 1914: 313

(http://hol.osu.edu/spmlnfo.html?id=MZSP\%2056467)

Holotype: ơ (\# MZSP 56467) '[Brazil], [São Paulo], São Paulo, 18.714 [Brazil, São Paulo, 1912, Barbiellini col.]' 'Pepsis coronaria Br.' 'Holotype, o', Pepsis coronaria Brè., det. C.R. Vardy, 1989" Pepsis chrysoptera Burm., o', det. C.R. Vardy, 1989'.

Current status: Pepsis chrysoptera Burmeister, 1872. 


\section{Pepsis erecta Brèthes, 1914: 299}

(http://hol.osu.edu/spmlnfo.html?id=MZSP\%2056533)

Holotype: ơ (\# MZSP 56533) '[Brazil], Bahia, V.[ila] Nova, 1908, Garbe leg,' 'Pepsis erecta Br, Brèthes det.17' 'Holotype, Pepsis erecta Brè, o', det. C.R. Vardy, 1989' 'Pepsis viridis Lep., o', det. C.R. Vardy, 1989'.

Current status: Pepsis viridis Lepeletier, 1845.

\section{Pepsis fuscorubra Brèthes, 1914: 321}

(http://hol.osu.edu/spmlnfo.html?id=MZSP\%2056468)

Holotype: 9 (\# MZSP 56468) ‘[Brazil], Goyaz [Goiás], 6.050 [Brazil, Goiás, 11.xi.1906, Baer col.]' Pepsis fuscorubra Br., Brèthes det. 17' 'Lectotype, 9 , Pepsis fuscorubra Bré., det. C.R. Vardy, 1988',

Current status: Pepsis terminata Dahlbom, 1843.

Comments: Vardy (2002: 68) has designated the specimen as a lectotype, however, according to original description the specimens listed herein is a holotype by monotypy.

\section{Pepsis garbei Brèthes, 1914: 297}

(http://hol.osu.edu/spmlnfo.html?id=MZSP\%2056497)

Lectotype: ㅇ (\# MZSP 56497) '[Brazil], Espírito Santo, 9.309 [Brazil, Espírito Santo, 1906, E. Garbe col.]' 'Pepsis garbei Brèthes, Brèthes det. 17.'Lectotype, \&, Pepsis garbei, Brèthes = viridis Lep., det. C.R. Vardy, 1991'.

Paralectotypes: 2ㅇ (\# MZSP 56498, 56499) '[Brazil], Espírito Santo, 3.537[Brazil, Espírito Santo, 1906, E. Garbe col.]' 'Pepsis garbei Br., Brèthes det. 17.' 'Paralectotype, + Pepsis garbei Brè. = viridis Lep., det. C.R. Vardy, 1991'.

Current status: Pepsis viridis Lepeletier, 1845.

Comments: Lectotype designated byVardy, 2005:248. The original publication listed incorrectly the number 3.537 as 3.573. The type series is composed by different species being the paralectotype (\# MZSP 56505) female with the following labels identified as Pepsis festiva Fabricius, 1804 '[Brazil], São Paulo, Raiz da Serra, xii, 18.523[Brazil, São Paulo, Estação Raiz da Serra, xii.1907, H. Lüderwaldt col.]' 'Pepsis garbei Br, Brèthes, det. 17.'PParalectotype, ’, Pepsis garbei Brè. = festiva F., det. C.R. Vardy, 1991'.

\section{Pepsis heterochroa Brèthes, 1914: 298}

(http://hol.osu.edu/spmlnfo.html?id=MZSP\%2056473)

Holotype: ㅇ (\# MZSP 56473) '[Brazil], Espírito Santo, 3.461 [Brazil, Espírito Santo, 1905, E. Garbe col.]' 'Pepsis heterochroa Bre., Brèth. Det. 17' 'Holotype, o, Pepsis heterochroa Brè., det. C.R. Vardy, 1989' 'Pepsis cassiope Mocs. Det. C.R. Vardy 1989'.

Current status: Pepsis cassiope Mocsáry, 1888.
Pepsis hora Brèthes, 1914: 289

(http://hol.osu.edu/spmlnfo.html?id=MZSP\%2056470)

Lectotype: + (\# MZSP 56470) '[Brazil], São Paulo, S.[ão] Sebastião, Bicego det.' 'Pepsis hora Br., Brèthes det. 17' 'Lectotype, + , Pepsis hora Brè. = festiva F., det. C.R. Vardy, 1991'.

Paralectotypes: 19 (\# MZSP 56472) '[Brazil], Espírito Santo, 3.444 [Brazil, Espírito Santo, x.1905, Garbe col.]' 'Pepsis hora Br, Brèthes det. 17' 'Paralectotype, + , Pepsis hora Brè. = festiva F., det. C.R. Vardy, 1991'; 1 \% (\# MZSP 56471) '[Brazil], São Paulo, São Sebastião, ix.[19]96" Pepsis hora $\mathrm{Br}$, 9 , Brèthes det. 17' 'Paralectotype, $\$$, Pepsis hora Brè. = festiva F., det. C.R. Vardy, 1991'.

Current status: Pepsis festiva Fabricius, 1804.

Comments: Lectotype designated by Vardy (2005: 219).

Pepsis humeralis Brèthes, 1914: 294

(http://hol.osu.edu/spmlnfo.html?id=MZSP\%2056469)

Holotype: ơ' (\# MZSP 56469) '[Brazil], São Paulo, Alto da Serra, xi, 16.227 [Brazil, Parque Cajuru, xi.1911, E. Schwebel col.]' 'Pepsis humeralis Br., Brèthes, det. 17' 'Holotype, o', Pepsis humeralis Brè., det. C.R. Vardy, 1991'.

Current status: Pepsis convexa Lucas, 1895.

Pepsis iheringi Brèthes, 1908: 238

(http://hol.osu.edu/spmlnfo.html?id=MZSP\%2056496)

Holotype: ㅇ (\# MZSP 56496) '[Brazil], Est.[ado] S.[ão] P.[aulo], Rincão, 12.382 [Brazil, São Paulo, Rincão]' 'Pepsis iheringi n. sp., Brèthes det. O'"Pepsis iheringi n. sp., Brèthes' 'Holotype, ${ }^{\prime}$, Pepsis iheringi Brè., det. C.R. Vardy, 1989' 'Pepsis smaragdina Dhlb., + , det. C.R. Vardy, 1989'.

Current status: Pepsis smaragdina Dahlbom, 1843.

Pepsis impatiens Brèthes, 1914: 273

(http://hol.osu.edu/spmlnfo.html?id=MZSP\%2056474

Holotype: ơ' (\# MZSP 56474) '[Brazil], São Paulo, Ypiranga [Ipiranga, neighborhood], i[january], 18.528 [Brazil, São Paulo, Ipiranga, i.1908, H. Lüderwaldt col.]' 'Pepsis impatiens Br., Breth. det. 17.' 'Holotype, Pepsis impatiens Brè., det. C.R. Vardy, 1989'.

Current status: Pepsis nitida Lepeletier, 1845.

\section{Pepsis inbio Vardy, 2000: 73}

Paratypes: 19 and 10' (\# MZSP 56475, 56476) 'Costa Rica, Guanacaste Prov.[ince], Fca. Jenny 31 km N Liberia, 300 m, Sep.[tember] 1988, GNP Biodiversity Survey, W85 34'27" N10 51'55"' 'Paratype, ơ, Pepsis inbio n. sp., det. C.R. Vardy, 1990'. 


\section{Pepsis limbatica Brèthes, 1914: 342}

(http://hol.osu.edu/spmlnfo.html?id=MZSP\%2056477)

Holotype: $\sigma^{7}$ (\# MZSP 56477) '[Brazil], Paraná, Palmeira, 12.413 [Brazil, Paraná, Palmeira, v.1898, Bisego col.]' 'Pepsis limbatica Br., Brèthes det. 17.' 'Holotype, o', Pepsis limbatica Brè., det. C.R. Vardy, 1989'"Pepsis limbatella Brè., o', det. C.R. Vardy, 1989'.

Current status: Pepsis flavescens Lucas, 1895.

\section{Pepsis marthae Vardy, 2002: 82}

Paratype: 19 (\# MZSP 56478) '[Peru], Chanchamayo [valley]' 'Chanchazaya [Chanchamayo], H. Rolle, Berlin col.' '2.741 [Peru, Chanchamayo, H. Rolle, Berlin col]' '99174' 'Paratype, + , Pepsis marthae, Vardy'.

\section{Pepsis martini Vardy, 2005: 260}

Paratypes: 10' (\# MZSP 56483) '[Brazil], S[ão] Paulo Ypiranga [Ipiranga, neighborhood], 2.iv.[1]936, Lange de Morrets col.' 'Paratype' 'Paratype, $\sigma^{x}$, Pepsis martini n. sp., Det. Vardy 2001'; $1 \sigma^{\top 1}$ (\# MZSP 56480) '[Brazil], Minas [Gerais], Pouso Alegre, xii.1953' Paratype, o', Pepsis martini n. sp., Det. Vardy 2001'; $10^{\top 1}$ (\# MZSP 56487) 'Brasil, São Paulo, Monte Alegre, 750 ms. Faz.[enda] Experimental, 14-27.x.1942, L. Trav. \& Almeida [col.]' 'Paratype, o’, Pepsis martini n. sp., Det. Vardy 2001'; 10" (\# MZSP 56481) '[Brazil], S.[ão] P.[aulo], Campinas, Guará, 20.xi.[19]88, R.P. Martins col.' 'Paratype, o', Pepsis martini n. sp., Det. Vardy

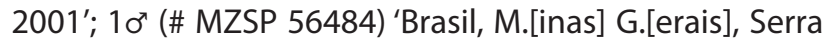
Caraça - 1380 m, xi.[1]961, Kloss, Lenko, Martins \& Silva col.' 'Paratype, ơ', Pepsis martini n. sp., Det. Vardy 2001'; $10^{7}$ (\# MZSP 56479) '[Brazil], Goyaz [Goiás], Leop.[oldo] Bulhões, xii.[19]33, Spitz [col]' 'Paratype, o', Pepsis martini n. sp., Det. Vardy 2001'; $10^{7}$ (\# MZSP 56485) '[Brazil], São Paulo, Campinas, 3.484 [Brazil, São Paulo, Campinas, 1906]' 'Pepsis dimidiata F., Brèthes det. 17' 'Paratype, o', Pepsis martini n. sp., Det. Vardy 2001'; $10^{\prime \prime}$ (\# MZSP 56486) 'Brasil, S.[ão] P.[aulo], Campinas, 15.xi.1900, A. Hempel col.' '6.502 [Brazil, São Paulo, Campinas, 15.xi.1900, A. Hempel col.]' ‘99398' ‘Paratype, Pepsis martini n. sp., Det. Vardy 2001'; 10" (\# MZSP 56482) '[Brazil], S.[ão] P.[aulo], Salesópolis, Est.[rada] Biol.[ógica] Boraceia, $850 \mathrm{~m}$, 5.iv.1966, Rabello col.' 'Paratype, o', Pepsis martini n. sp., det. Vardy 2001'.

\section{Pepsis militaris Brèthes, 1914: 343}

(http://hol.osu.edu/spmlnfo.html?id=MZSP\%2056502)

Lectotype: 9 (\# MZSP 56502)‘[Brazil], São Paulo, [Campos do] Jordão, ii.[19]06, Lüd.[erwaldt] [col.]' 'Lectotype' 'Pepsis militaris Br. Brèth. Det. 1.'Lectotype, \&, Pepsis militaris Brè. = flavescens Luc., det. C.R. Vardy, 1991'.

Paralectotypes: 29 (\# MZSP 56503, 56504) '[Brazil], São Paulo, [Campos do] Jordão, ii.[19]06, Lüd.[erwaldt] [col.]' 'Pepsis militaris Br. Brèth. Det. 17.'Lectotype,,+ Pepsis militaris Brè. = flavescens Luc., det. C.R. Vardy, 1991'.
Current status: Pepsis flavescens Lucas, 1895.

Comments: Lectotype designated by Vardy (2005: 176).

Pepsis minarum Brèthes, 1914: 286

(http://hol.osu.edu/spmlnfo.html?id=MZSP\%2056531)

Holotype: 9 (\# MZSP 56531) ‘[Brazil], Minas Gerais, Irará, 12.414 [Brazil, Minas Gerais, Irará, Dr. Bach col.]' 'Pepsis minarum Br., Brèthes det. 17' 'Lectotype, Pepsis minarum Brè., det. C.R. Vardy, 1988'.

Comments: Vardy (2005: 140) has designated the specimen as a lectotype, however, according to the original description the specimen listed herein is a holotype by monotypy.

\section{Pepsis nanoides Vardy, 2005: 268}

Paratypes: 19 (\# MZSP 56488) '[Brazil], São Paulo, Rio Claro, Horto Florestal [Edmundo] Navarro de Andrade, 30.vi.[19]87, coletado no barranco, R.P. Martins, col.' 'Paratype, , , Pepsis nanoides n. sp., Det. Vardy, 2001'; 1 ? (\# MZSP 56489) '[Brazil], Pará, Belém, June, 1924' 'F.X. Williams collector' 'Paratype, + , Pepsis nanoides n. sp., Det. Vardy, 2001'; 19 (\# MZSP 56490) '[Brazil], E.[stado] de S.[ão] Paulo, 12.430 [Brazil, São Paulo, Jundiaí, 21.iv.1899, M. Beron col.], Pepsis dimidiata' 'Head Figured Specimen' 'Paratype, , Pepsis nanoides n. sp., Det. Vardy 2001' 'MZUSP'; 1 o (\# MZSP 56491) '[Brazil], E[stado] S[ão] Paulo, Jundiahy [Jundiaí],,+ 12.430 [Brazil, São Paulo, Jundiaí, 21.iv.1899, M. Beron col.], Pepsis dimidiata' 'Coll antiga' 'Pepsis dimidiata Fabr.' 'Paratype, 9 , Pepsis nanoides n. sp., Det. Vardy, 2001"MZUSP'; 1 o (\# MZSP 56492) 'Brasil, S.[ão] P.[aulo], Itu, Faz.[enda] Pau D'Alho, ii.1963, F. Werner \& U. Martins col.' Pepsis dimidiata, Fabr., + , det. H. Evans 1967' 'Pepsis dimidiata, Fabr.' Paratype,,+ Pepsis nanoides n. sp., det. Vardy 2001'.

Comments: The original publication listed the specimen from Belém (Brazil) (\# MZSP 56489) as collected on May, but the correct date in the label is June.

\section{Pepsis pacifica Brèthes, 1914: 326}

(http://hol.osu.edu/spmlnfo.html?id=MZSP\%2056493)

Holotype: + (\# MZSP 56493) 'Bolívia, Yungas [de La Paz], 1000 m., det. P' 'Pepsis pacifica Br., Brèthes det. 17.' 'Holotype, +, Pepsis pacifica Brè., det. C.R. Vardy, 1989' 'Pepsis montezuma Sm., + , det. Vardy, 1987'.

Current status: Pepsis montezuma Smith, 1855.

Pepsis paulistana Brèthes, 1914: 307 (http://hol.osu.edu/spmlnfo.html?id=MZSP\%2056466)

Holotype: ơ' (\# MZSP 56466) '[Brazil], São Paulo, [São Paulo], Ypiranga [Ipiranga, neighborhood]' 'Pepsis paulistana Brèthes det. 17 Brithey' 'Holotype, o', Pepsis pau- 
listana Bre., det. C.R. Vardy, 1989' 'Pepsis chrysoptera Burm., o", det. C.R. Vardy, 1989'.

Current status: Pepsis chrysoptera Burmeister, 1872.

Pepsis pulchra Brèthes, 1914: 323

(http://hol.osu.edu/spmlnfo.html?id=MZSP\%2056532)

Holotype: $\sigma^{\top}$ (\# MZSP 56532) '[Brazil], São Paulo, Jundiahy [Jundiaí], Beron leg.' 'Lectotype' 'P. pulchra Brè., det. C.R. Vardy, 1988' 'Pepsis minarum Brè., det. C.R. Vardy, 1989'.

Current status: Pepsis minarum Brèthes, 1914.

Comments: Vardy (2005: 140) has designated the specimen as a lectotype, however, according to original description the specimen listed herein is a holotype by monotypy.

\section{Cryptocheilus Panzer}

Salius diffusus Brèthes, 1911: 67

(http://hol.osu.edu/spmlnfo.html?id=MZSP\%2056534)

Holotype: 9 (\# MZSP 56534) '[Brazil, São Paulo, São Paulo], Ypiranga [Ipiranga, neighborhood], \$, 2.013 [Brazil, São Paulo, São Paulo, Ipiranga, J. Lima col.]' 'Salius diffusus, Brèthes det.' 'Salius diffusus, Brèthes 1911, \&, Holotypus, A. Roig Alsina exam. 1981' Salius diffusus' 'R. Wahis rv. 86, Holotype, Salius diffusus, Breth, 1911, $9=$ Entypus cyaneus, Lep. 1845, O'R. Wahis dt. 86, Entypus cyaneus cyaneus, Lep.'

Current status: Cryptocheilus diffusus (Brèthes,1911)

Salius (Cyphononyx) notatipennis Fox, 1899: 196 (http://hol.osu.edu/spmlnfo.html?id=MZSP\%2056501)

Holotype: \& (\# MZSP 56501) '[Brazil], Rio Grande do Sul' 'Salius (Cyphornonyx) notatipennis, Fox, Type, 12.482[Brazil, Rio Grande do Sul], ᄋ, Fox det.' Holotypus, Salius (Cyphononyx) notatipennis Fox, 1899, A. Roig Alsina Glam., 1981"Salius (Cyphononyx) notatipennis, Fox"'Salius natatipennis Fox = Anacyphonyx notatipennis (Fox), det. A. Rog. Alsina, 1982'.

Current status: Cryptocheilus notatipennis (Fox, 1899)

\section{THYNNOIDEA THYNNIDAE \\ Myzininae \\ Mizinini \\ Pterombrus Smith}

Pterombrus iheringi Ducke, 1914: 117

(http://hol.osu.edu/spmlnfo.html?id=MZSP\%2056536)

Holotype: + (\# MZSP 56536) '12.446 [Brazil, São Paulo, São Paulo, Ipiranga, J. Lima coll.], Ypiranga [Ipiranga, neighborhood], typo, Ducke det.' 'Pterombrus iheringi, Ducke, typo' 'Holotipo' 'Pterombrus iheringi, Ducke'.

\section{Huberia glabricollis Ducke, 1907a: 06}

Syntype: 19 (\# MZSP 56537) 'Brazil, Estado do Pará' '16.624[Brazil, Pará, Parinha, v.1903], Cotypo' 'Pterombrus glabricollis Ducke, cotypo"Prainha, 10.v.1903 Ducke"cótipo"'Pterombrus glabricollis Ducke'.

Current status: Pterombrus glabricollis (Ducke, 1907).

\section{Thynninae \\ Rhagigasterini \\ Aelurus Klug}

\section{Aelurus grandis Kimsey, 1991 b: 234}

Paratype: 19 (\# MZSP 56538) '[Brazil], S.[ão] Paulo, C.[ampos do] Jordão, i.1948' 'Paratype Aelurus grandis, L.S. Kymsey'.

\section{Elaphropterini Atopothynnus Kimsey}

\section{Atopothynnus unidens Kimsey, 1991a: 73}

Paratypes: $10^{7}$ and 19 (\# MZSP 57213) on the same pin '[Brazil], Paraná, Curitiba, vi.[1]937, P. Pereira [col]' 'Paratype Atopothynnus unidens L.S. Kinmsey'.

\section{Elaphroptera Guerin-Meneville}

\section{Elaphroptera montifacies Genise \& Kimsey, 1993: 209}

Paratypes: 10' (\# MZSP 57214) 'Brazil, Santa Catarina, Nova Teutônia, ix.1964, Fritz Plaumann [col]' 'Paratype Elaphroptera montifacies Genise \& Kimsey'; $10^{\top 7}$ (\# MZSP 57215) same data except '11.[19]66'.

\section{Glottynoides Kimsey}

\section{Glottynoides genisei Kimsey, 1991: 61 in Genise \&} Kimsey, 1991

Paratype: 10' (\# MZSP 56880) 'Argent[ina], Cordoba, Carlos Paz - Capilla del monte, 26.Feb.[19]66, C.C. Porter' 'Paratype Glottynoides genisei L.S. Kimsey'.

\section{Merithynnus Kimsey}

\section{Merithynnus paradoxus Kimsey, 2005: 591}

Paratype: 10' (\# MZSP 56879) 'Colombia, Norte de Santander PNN [Parque Nacional Natural], Tamá, El 
Arenal, $07^{\circ} 25^{\prime} \mathrm{N}, 72^{\circ} 26^{\prime} \mathrm{W}$, Malaise 7-15.viii.1998, A. Cortés Leg. M.291.'’Paratype Merithynnus paradoxus Kimsey'.

\section{Ornepetes Guerin-Meneville}

\section{Ornepetes sylvicola Bradley, 1911: 131}

Paratypes: 10" (\# MZSP 57211) 'Brazil, State Sao Paulo, Hammar coll.' 'Cornell U. Lot 298. sub 111'20.871 [Estado de São Paulo, Hammar col.]' 'Paratype Ornepetes sylvicola, J.C. Bradley' 'Ornepetes sylvicola Bradley' '99841' 'Sao Paulo'; 10' (\# MZSP 57212) same data except 'sub 43' ‘99842'J.C. Bradley Dissection No. 52'.

\section{Upa Kimsey}

Upa bidentata Kimsey, 1996: 57

(http://hol.osu.edu/spmlnfo.html?id=MZSP\%2056539)

Holotype: $\sigma^{\rtimes}$ (\# MZSP 56539) ‘Brazil, Santa Catarina, Nova Teutonia, x.1964, Fritz Plaumann [col.]' 'Holotype Upa bidentata Kimsey'.

Paratype: 10' (\# MZSP 56540) '[Brazil], Paraná, Curityba[Curitiba], 4.i.[19]37, C. Wsterman coll.'Paratype Upa bidentata LS Kimsey'.

\section{Upa longispina Kimsey, 1996: 59}

(http://hol.osu.edu/spmlnfo.html?id=MZSP\%2056541)

Holotype: o' (\# MZSP 56541) '[Brazil], S.[ão] Paulo, H.[orto] Florestal' 'John Lane Collector' 'Holotype Upa longispina Kimsey'.

\section{Upa nasuta Kimsey, 1996: 60}

Paratypes: 20' (\# MZSP 56542, 56543) 'Brazil, S.[ão] P.[aulo], S.[ão] J.[osé do] Barreiro, Serra da Bocâina, 13-17.i.1969, Porter, García [col.]' ‘Paratype Upa nasuta Kimsey'; 10' (\# MZSP 56544) 'Brazil, São Paulo, Serra [da] Bocaina, 13-17.i.1969'.

\section{Upa tridentata Kimsey, 1996: 62}

(http://hol.osu.edu/spmlnfo.html?id=MZSP\%2056545)

Holotype: $\sigma^{\top}$ (\# MZSP 56545) 'Br[azil], Rio de Janeiro, Mury, Nova Friburgo, xii.1975, Gred \& Guimarães col' 'Holotype Upa tridentata Kimsey'.

Paratype: 107 (\# MZSP 57209) same data as holotype, except 'xii.1976' and additional labels ' $q$ is species of Dolichothynnus det L.S. Kimsey' 'Paratype Upa tridentata Kimsey'.

Comments: Additionally to the specimens listed, there is $10^{\text {' }}$ (\# MZSP 57210) with the following label data 'S[ão] P[aulo], Jaraguá, 9.xii.1962, J.D. Cardoso col.' 'Paratype Upa tridentata Kimsey' that are not cited in the original publication although it has the paratypes labels from L.S. Kimsey.

\section{VESPOIDEA \\ VESPIDAE \\ Eumeninae \\ Hypalastoroides Saussure}

Alastor rotundiceps Schrottky, 1911: 19

Syntype: 19 (\# MZSP 57085) 'Paraguay, V[illa] Encarnacion, Alastor rotundiceps Schrottky [col.], 16.344 [Paraguay, Encarnacion, Schorttky col.], Schrottky det.' '16.344 [Paraguay, Encarnación, i.1906, Schrottky col.]' 'Alastor rotundiceps $\$$ Schrottky, cotype, C. Alastor Schrottky det. 1910' 'Lectotype of Hypalastoroides rotundiceps (Schr.) des. by J.V.D. Vecht 1981'.

Current status: Hypalastoroides rotundiceps (Schrottky, 1911).

Comments: The specimen has a lectotype label from Jacobus van der Vecht, however there is no a published designation of this lectotype.

\section{Polistinae \\ Agelaia Lepeletier}

Stelopolybia angulata bertonii Richards, 1978: 244

Paratype: 19 (\# MZSP 57101) '[Brazil], S.[anta] Catharina [Catarina], Joinville, 17.018 [Santa Catarina, Joinville, comp. Rolle col., xi.1900]' 'Stelopolybia angulata Ducke rev. 11' S. angulata ssp. bertonii Rich., + paratype'.

Current status: Agelaia angulata (Fabricius, 1804).

Comments: In addition to the specimens listed, the original publication reported one female from Espírito Santo (Brazil) deposited at MZSP, however, this paratype was not found in the colletion.

\section{Stelopolybia cornelliana subterranea Richards, 1978: 242}

Paratypes: 29 (\# MZSP 57099-57100) 'Peru, Tomentoy, canyn base of Machu Pichu, 2000 m, 19-24.vi.64, (B. Malkin)' 'Stelopolybia cornelliana Rich. ssp. subterranea Rich., + , paratype'.

Current status: Agelaia cornelliana subterranea (Richards, 1978).

Comments: In addition to the specimens listed, the original publication reported one more female paratype deposited at MZSP, however, these specimens were not found in the colletion. 


\section{Stelopolybia hamiltoni Richards, 1978: 251}

Paratypes: 2 9 (\# MZSP 57096, 57097) '[Brazil], E.[stado] Amaz.[onas], Rio Juruá, 2.164 [Amazonas, Rio Juruá, Garbe leg.]' 'Stelopolybia vulgaris, Ducke rev. 11' 'Stelopolybia hamiltoni Rich., ㅇ, paratype'.

Current status: Agelaia hamiltoni (Richards, 1978).

Comments: In addition to the specimens listed, the original publication reported more two females paratypes from Amazonas (Brazil) and one female paratype from Rondônia (Brazil) deposited at MZSP, however, these paratypes were not found in the colletion.

\section{Stelopolybia lobipleura Richards, 1978: 247}

Paratype: 19 (\# MZSP 57098) ‘Bras.[il], Mato Grosso, Serra [do] Roncador, R.S. Base Camp., 7.ix.68' 'W. Hamilton col.' 'Stelopolybia n. sp.' 'Stelopolybia lobipleura Rich.,, , paratype'.

Current status: Agelaia lobipleura (Richards, 1978).

\section{Apoica Lepeletier}

\section{Apoica (Apoica) strigata Richards, 1978: 267}

Paratypes: 19 and 10' (\# MZSP 56881, 56882) 'Brasil, Amazonas, Benjamin Constant, 18-28.ix.1962, K. Lenko - col.' 'Apoica strigata Rich., paratype'; 30' (\# MZSP 56883-56885) '[Brazil], AM [Amazonas], Tapuruquara, Rio Negro, 26-27.xi.1962, J. Bechyné col.' 'Apoica strigata Rich., on', paratype'.

\section{Brachygastra Perty}

\section{Nectarinia baccalaurea lhering, 1903: 154}

(http://hol.osu.edu/spmlnfo.html?id=MZSP\%2056886)

Lectotype: + (\# MZSP 56886) 'Perú, 827 [Peru, Stand col.]' 'Nectarinia baccalaurea R. v. Ih., Ducke rev. 11' 'Brachygastra baccalaurea (R. Von lhering), det. M. Naumann" Nectarinia baccalaurea R. v. Iher., o, lectotype'.

Current status: Brachygastra baccalaurea (Ihering, 1903).

Comments: Lectotype designated by Richards (1978: 177).

\section{Brachygastra fistulosa Naumann, 1968: 949}

Paratypes: 19 (\# MZSP 56888) 'Brasil, 830' 'Paratype, Brachygastra fistulosa, M.G. Naumann' 'Nect. aug. var. quinta R.v.lh., R.v.lh rev. 12'; 19 (\# MZSP 56889) 'Brazil, 830' 'Paratype, Brachygastra fistulosa, M.G. Naumann' 'Nectarinia augusti var. quinta Sauss, R.v.lh., Ducke rev. 1911 '.
Brachygastra mouleae Richards, 1978: 170

(http://hol.osu.edu/spmlnfo.html?id=MZSP\%2056906)

Holotype: + (\# MZSP 56906) 'Brazil, S.[ão] P.[aulo], Rio Claro, Faz.[enda] Jussara, Nest, 4.ix.69, V.M. Rodrigues' 'Brachygastra mouleae Rich., , holotype'.

Paratypes: 19 (\# MZSP 56907) 'Brazil, S.[ão] P.[aulo], Rio Claro, Nest, 4.ix.69, V.M. Rodrigues' 'Brachygastra mouleae Rich., \&, paratype'; 19 (\# MZSP 56908) 'BR [Brazil], S.[ão] P.[aulo], R.[io] Paraná, Porto Cabral [Right bank of the Paraná River], 1-25.iv.1944, Trav. Fo. \& Carrera \& E. Dente" 'Brachygastra augusti (Sauss.), det. M. Naumann 1965' 'Brachygastra mouleae Rich., ㅇ, paratype'.

Comments: In addition to the specimens listed, the original publication reported one female from Rio Claro (São Paulo, Brazil) deposited at MZSP, however, this paratype was not found in the colletion.

\section{Caba lhering}

Caba bilineolata var. fasciata lhering, 1904: 112 (http://hol.osu.edu/spmlnfo.html?id=MZSP\%2056887)

Lectotype: 9 (\# MZSP 56887) 'Surinam[e], 785 [Suriname, Stand col.]' 'Nect. 2-lin. Spin. var. fasciata R. v. Ihg., R. v. Ihg. det.' 'Nectarina 2-lineolata Spin., Ducke det. 11' 'Brachygastra bilineolata var. fasciata (R.V. Iher.), ๆ, lectotype'.

Current status: Caba fasciata lhering, 1904.

Comments: Lectotype designated by Richards (1978: 171).

\section{Chartergellus Bequaert}

Chartergellus amazonicus Richards, 1978: 220

Paratype: 19 (\# MZSP 56891) 'Brasil, Amazonas, Benjamin Constant, 18-28.ix.1962, K. Lenko - col.'Chartergellus amazonicus Rich., $\$$, paratype'.

\section{Chartergellus nigerrimus Richards, 1978: 222}

Paratype: 19 (\# MZSP 56892) 'Peru, 16.734 [Peru, 1905, Stand. col.]'Parachart. compressus, Ducke rev. 11' '99502' 'Chartergellus nigerrimus Rich.,,+ paratype'.

\section{Chartergellus punctatior Richards, 1978: 221}

Paratype: 19 (\# MZSP 56893) '[Brazil], Estado do Pará, 16.400 [Pará, xi.1899, Ducke col.]' 'Parachartergus fronta-

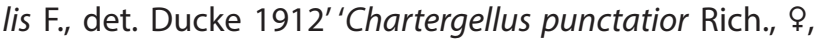
paratype'. 


\section{Clypearia Saussure}

\section{Clypearia duckei Richards, 1978: 196}

Paratypes: 19 (\# MZSP 56894) 'Bras.[il], Acre, Cruzeiro do Sul, 26.11.[19]68, Ant complex, Nov[ember] 78' 'W. Hamilton col.' 'Clypearia n. sp.' 'Clypearia duckei Rich., + , paratype'; 19 (\# MZSP 56895) 'Brasil, Pará, Canindé (Rio Gurupí), x.1964, B. Malkin col.' 'Clypearia duckei Rich., + , paratype'.

\section{Metapolybia Ducke}

\section{Metapolybia miltoni Andena \& Carpenter 2011: 117} (http://hol.osu.edu/spmlnfo.html?id=MZSP\%2056896)

Holotype: + (\# MZSP 56896) 'Brasil, S.[ão] P.[aulo], Ribeirão Preto, $21^{\circ} 09^{\prime} \mathrm{S}, 47^{\circ} 51^{\prime} \mathrm{W}, 860$ m, 15 may 1997, James M. Carpenter Nest, 970515-9' Metapolybia miltoni, Antena \& Carpenter'.

Paratypes: 19 (\# MZSP 56897) 'Brasil, S.[ão] P.[aulo], Ribeirão Preto, $21^{\circ} 09^{\prime} \mathrm{S}, 47^{\circ} 51^{\prime} \mathrm{W}, 860$ m, 15 may 1997, James M. Carpenter Nest 970515-9' 'Metapolybia miltoni, Antena \& Carpenter'; 19 (\# MZSP 56898) 'Brazil, S.[ão] P.[aulo], Ribeirão Preto, $21^{\circ} 09^{\prime} \mathrm{S}, 47^{\circ} 51^{\prime} \mathrm{W}, 860 \mathrm{~m}, 15$ may 1997, James M. Carpenter Nest, 970515-9' 'Metapolybia docilis Richards, det. J.M. Carpenter 1997' 'Metapolybia miltoni Andena \& Carpenter'.

\section{Parachartergus Ihering}

\section{Parachartergus bentobuenoi lhering, 1904}

(http://hol.osu.edu/spmlnfo.html?id=MZSP\%2091838)

Lectotype: 9 (\# MZSP 91838) '[Brazil], E.[stado] Amaz. [onas], Rio Juruá, 1576 [x.02, Amazonas, Rio Juruá] 'Parachart. bentob. R. v. Ih., R. v. Ih. Rev. 12.' 'Lectotype, $\uparrow$, of Parachartergus bentobuenoi, R. v. Ihering 1904, Richards, 1971'

\section{Current status: Parachartergus griseus Fox, 1898}

Comments: Lectotype designated by Richards (1978: 213)

\section{Parachartergus lenkoi Richards, 1978: 213}

(http://hol.osu.edu/spmlnfo.html?id=MZSP\%2056899)

Holotype: 9 (\# MZSP 56899) '[Brazil], M.[ato] G.[rosso], Utiariti, Rio Papagaio, 30.x.1966, Lenko \& Pereira' 'KL.161' 'Parachartergus lenkoi Rich., holotype, ᄋ'.

Paratypes: 19 (MZSP 61955) '[Brazil], MT [Mato Grosso], Utiariti, Rio Papagaio, x.1966, Lenko \& Pereira' 'Parachartergus griseus (Fox)' 'Parachartergus lenkoi, Det. J. M. Carpenter'; 1o (MZSP 61954) '[Brazil], MT [Mato Grosso], Utiariti, Rio Papagaio, xi.1966, Lenko \& Pereira';
19 (MZSP 62027) '[Bolivia], [La Paz], Yungas Camp. Sacramento, Ingavi - Coroico, 2500m, 9-11.vii.[19]64, B. Malkin' 'Parachartergus lenkoi, Det. J. M. Carpenter'; 117 ? (MZSP 56899-56905; 61897-61953;61967-62026)'[Brazil], MT [Mato Grosso], Utiariti, Rio Papagaio, 30.x.1966, Lenko \& Pereira' 'KL 161'; 6 9 (\# MZSP 56900-56905) '[Brazil], Utiariti, MT [Mato Grosso], Rio Papagaio, 30.x.1966, Lenko \& Pereira"KL.161"'Parachartergus lenkoi Rich., +, paratype'.

\section{Polybia Lepeletier}

\section{Polybia dubitata Ducke, 1910: 500}

(http://hol.osu.edu/spmlnfo.html?id=MZSP\%2056909)

Lectotype: 9 (\# MZSP 56909) 'Brazil, Estado do Pará' 'Est. [ado] Pará, Óbidos, 16.417 [Pará, Óbidos, 1912, Ducke col.]'Polybia dubitata Ducke, 1910, \&, lectotype'.

Current status: Polybia procellosa Zavattari, 1906

Comments: Lectotype designated by Richards (1978: 126).

\section{Polybia furnaria lhering, 1904: 217}

(http://hol.osu.edu/spmlnfo.html?id=MZSP\%2056890)

Lectotype: @ (\# MZSP 56890) '[Brazil], Est.[ado] Pará, Santarém, 1.571 [Pará, Santarém, E. Garbe col.]' 'Polybia furnaria, Ducke ver. 11' Polybia furnaria R. v. Ih., ㅇ, lectotype'.

Comments: Lectotype designated by Richards (1978: 121).

\section{Polybia incerta Ducke, 1907b: 179}

Paralectotype: 19 (\# MZSP 57086) 'Brazil, Estado do Amazonas' 'Teffé [Tefé], 7.xi.1904, Ducke' '16.418 [Amazonas, Tefé, xi.1904, Ducke col.]' 'Polybia incerta Ducke, + , paralectotype'.

Comments: Paralectotype designated by Richards, 1978: 124. The lectotype from Amazonas (Tefé) was designated by Richards (1978: 124) and indicated by him as deposited at MZSP, however, it was not found in the collection.

\section{Polybia (Myrapetra) platycephala sylvestris Richards,} 1978: 91

Paratypes: 19 (\# MZSP 56910) 'Brasil, Terr.[a] Amapá, Rio Felício, 2.vii.1959, J. Lane leg.' 'Coleção J. Lane'; 1 ( (\# MZSP 56911) '[Brazil, Amazonas], Rio Parauary [Parauari], 3[iii].1937' 'Polybia platycephala R. ssp. sylvestris Rich., ㅇ, paratype'; 10 \% (\# MZSP 56912-56921) ‘[Brazil], MT [Mato Grosso], Utiariti, Rio Papagaio, 5.xi.1966, Lenko \& Pereira' 'Polybia platycephala sylvestris Rich., \$, paratype'; 39 (\# MZSP 56922-56924) '[Brazil], Est.[ado] Rio [de Janeiro], C.[ampo] Itatinga, 2.840 [Campo Itatinga, 
iv.1906, H. Luederwaldt col.]' 'P. platycephala sylvestris Rich., + , paratype'; 49 (\# MZSP 56925-56928) 'Brasil, São Paulo, Osasco, 9.xi.1956, M.A. Vulcano col.'; 19 (\# MZSP 57087) 'Brasil, S.[ão] Paulo, Ilha dos Búzios, 16.x - 4.xi. [1]963, Exp.[edição] Dep.[artamento] Zool.[ogia]'Polybia platycephala Rich. ssp. sylvestris Rich'.

Comments: In the original publication the collection date of the specimen \# MZSP 56911 was incorrectly transcribed as February. Additionally, the following paratypes were also cited in the original publication as deposited at MZSP but were not found: four females from Rio Parauary (Amazonas, Brazil), one female from Barra dos Tapirapés (Mato Grosso, Brazil), 93 females from Utiariti (Mato Grosso, Brazil), 19 females from Ilha dos Búzios (São Paulo, Brazil), 6 females from São Sebastião (São Paulo, Brazil), 5 females from Campos do Jordão (São Paulo, Brazil), and 3 females from Osasco (São Paulo, Brazil).

Polybia (Myrapetra) ruficeps xanthops Richards, 1978: 76 (http://hol.osu.edu/spmlnfo.html?id=MZSP\%2056929)

Holotype: 9 (\# MZSP 56929) '[Brazil], MT [Mato Grosso] [now Mato Grosso do Sul], Três Lagoas, marg.[em] esq. [uerda] rio Sucuriu, Faz.[enda] Canaã, iv. 1967, F. Lane col.' 'Polybia ruficeps Schrottky ssp. xanthops Rich., o, holotype'.

Paratypes: 19 (\# MZSP 56930) 'Brazil, BA [Bahia], Villa Nova, E. Garbe' '18.081 [Bahia, Villa Nova, E. Garbe col.]' 'Polybia ruficeps ssp. xanthops Rich., + , paratype'; 39 (\# MZSP 56931-56933) '[Brazil], Ceará, Quixaramobim, xi.1940' 'Polybia ruficeps Schrottky ssp. xanthops Rich., O, paratype'; 2 9 (\# MZSP 56934-56935) '[Brazil], MT [Mato Grosso] [now Mato Grosso do Sul], Três Lagoas, marg. [em] esq.[uerda] rio Sucuriu, Faz.[enda] Canaã, iv.1967, F. Lane col.''Poybia ruficeps Schrottky ssp. xanthops Rich., o, paratype'; 3 9 (\# MZSP 56936-56938) '[Brazil], MG [Minas Gerais], Piraborá [Pirapora], 1912, E. Garbe"17.374 [Minas Gerais, Pirapora, 1912, E. Garbe col.]' 'Polybia ruficeps Schorttky ssp. xanthops Rich., \&, paratype'.

\section{Polybia scutellaris var. paulista Ihering, 1896: 160 (http://hol.osu.edu/spmInfo.html?id=MZSP\%2057088)}

Lectotype: 9 (\# MZSP 57088) ‘[Brazil, São Paulo], Ypiranga [Ipiranga], Glattes Nest' 'Polybia scutellaris var. paulista $\mathrm{H}$. v. Iher., , lectotype'.

Current status: Polybia paulista Ihering, 1896.

Comments: Lectotype designated by Richards (1978: 85).

\section{Protopolybia Ducke}

\section{Protopolybia bituberculata Silveira \& Carpenter 1995: 48}

Paratypes: 39 (\# MZSP 57089-57091) 'Peru, Depto. [Departament] Loreto, 40 km NE Iquitos, 29 December
1990, Carpenter \& Wenzel, Nest 901229-7' 'Paratype, Protopolybia bituberculata, Silveira \& Carpenter'.

\section{Mischocyttarini Mischocyttarus Saussure}

\section{Megacanthopus alfkenii Ducke, 1904: 362 \\ (http://hol.osu.edu/spmlnfo.html?id=MZSP\%2056939)}

Lectotype: @ (\# MZSP 56939) 'Brasil, Guyana [now Amapá], Rio Villa Nova, 17.107 [Rio Villa Nova, Amapá, x.1900, Ducke col.]' 'Megacanth. indet. var. alfkenii, Cotypo!, Ducke rev 11.'Mischocyttarus (Megacanthopus) alfkenii (Ducke, 1904), lectotype, Richards, 1970'.

Comments: Lectotype designated by Richards (1978: 343).

Current status: Mischocyttarus (Megacanthopus) alfkenii (Ducke, 1904).

\section{Mischocyttarus archidonaensis Zikán, 1949: 43 (http://hol.osu.edu/spmlnfo.html?id=MZSP\%2057102)}

Holotype: 9 (\# MZSP 57102) ‘Ecuador, Archidona, 16.668 [Ecuador, Archidona]' 'Mischocyth. labiatus F., Ducke rev 11 ' Mischocyttarus archidonaensis Zik., J.F. Zikán determ.' 'Mischocyttarus archidonaensis Zik., \&, holotype'.

Current status: Mischocyttarus tomentosus Zikán, 1935.

\section{Mischocyttarus atramentarius Zikán, 1949: 206} (http://hol.osu.edu/spmlnfo.html?id=MZSP\%2056940)

Lectotype: ㅇ (\# MZSP 56940) '[Brazil, Rio de Janeiro], Itatiaya [Itatiaia] - 800 m km, Monts, Bau V, 5.xi.1931, J.F. Zikán', 'Mischocytt. atramentarius Zik., J.F. Zikán determ.' 'Mischocyttarius atramentarius Zik., o, lectotype, Richards 1970'.

Paralectotypes: 19 (\# MZSP 56946) '[Brazil] Est.[ado] do Rio [de Janeiro], Itatiaya [Itatiaia], 500 m Valparaízo, 17.ii.1933 c.I [?] Ban, J.F. Zikán' 'Mischocytt. atramentarius Zik., J.F. Zikán determ.' M. atramentarius Zik., o', paratype'; 10" (\# MZSP 56947) '[Brazil] Est.[ado] do Rio [de Janeiro], Itatiaya [Itatiaia], 500 m Valparaízo, 3.ii.1933, [?] Ban, J.F. Zikán' 'Mischocytt. atramentarius Zik., J.F. Zikán determ.' 'M. atramentarius Zik., o, paratype'; 1 ơ (\# MZSP 56948) '[Brazil, Rio de Janeiro], Itatiaya [Itatiaia], 550 m km, Faz. [enda] Valpar.[aízo], 25.ii.1933, J.F. Zikán' 'Mischocytt. atramentarius Zik., J.F. Zikán determ.' 'M. atramentarius Zik., ㅇ, paratype'; 19 (\# MZSP 56949) '[Brazil, Rio de Janeiro], Itatiaya [Itatiaia], $550 \mathrm{~m} \mathrm{~km}$, Faz.[enda] Valpar. [aízo], 17.ii.1933, J.F. Zikán"Mischocytt. atramentarius Zik., J.F. Zikán determ.' 'M. atramentarius Zik., o, paratype'; 10 " (\# MZSP 56950) ‘[Brazil] Est.[ado] do Rio, Itatiaya [Itatiaia] - 700 m, 14.ii.1932, Ban v, J.F. Zikán' 'Mischocytt. atramentarius Zik., J.F. Zikán determ.' 'M. atramentarius Zik., o', paratype'; 10" (\# MZSP 56945) '[Brazil, Rio de Janeiro], 
Itatiaya [Itatiaia], 800 m km, Monts, [?] Ban v, 5.xi.1931' 'Mischocytt. atramentarius Zik., J.F. Zikán determ.' 'M. atramentarius Zik., $\sigma^{*}$, paratype'; 29 and $10^{*}$ (\# MZSP 56941-56943) '[Brazil], E.[stado] S.[ão] Paulo, Ypiranga [Ipiranga], 1.052 [SãooPaulo, Ipiranga]' 'Megacanth. ater, Ducke rev. 11"'Mischocytt. atramentarius Z., J.F. Zikán determ.'M. atramentarius Zik. paratype'; 1 9 (\# MZSP 56944) '[Brazil], E.[stado] S.[ão] Paulo, Jundiahy [Jundiaí], 17.086 [São Paulo, Jundiaí, col. M. Beron' 'Megacanthop. ater, Ducke rev. 11' 'Mischocytt. atramentarius Zik., J.F. Zikán determ.'M. atramentarius Zik., $\$$, paratype'.

Comments: Lectotype designated by Richards (1978: 298).

\section{Mischocyttarus bahiaensis Zikán, 1949: 882}

(http://hol.osu.edu/spmlnfo.html?id=MZSP\%2056953)

Holotype: 9 (\# MZSP 56953) 'Brésil, Bahia, E. Mocquerys 1845, Museum Paris' '16.475 [Bahia, Coll. Museu Paris, R.v. Ihg], Buysson det., Megacanthopus surinamensis Sauss.' 'Mischocyttarus bahiansis Zik., J.F. Zikán determ.' 'Mischocyttarus bahiaensis Zik., \&, holotype, Richards, 1970'.

\section{Mischocyttarus capichaba Zikán, 1949: 83}

(http://hol.osu.edu/spmlnfo.html?id=MZSP\%2056951)

Holotype: (\# MZSP 56951) '[Brazil], Esp.[írito] Santo, Garbe leg. [19]06, 7.239 [Espírito Santo, E. Garbe col., Ducke rev.]"Mischocyttarus indeterminabilis Sauss, Ducke rev. 11' Mischocyttarus capichaba Zik., J.F. Zikán determ. 'Mischocittarus capichaba Zik., \&, holotype, Richards 1970'.

\section{Mischocyttarus carbonarius r. flavonotatus Zikán, 1949: 189 \\ (http://hol.osu.edu/spmlnfo.html?id=MZSP\%2056954)}

Holotype: 9 (\# MZSP 56954) 'Bolivia, Yungas de La Paz, 2.727 [Yungas de La Paz, Bolivia, 1903, col. H. Rolle, Berlin]"Holotypus' Megacanthop. flavisaus, Ducke rev. 11' 'Mischoc. flavicans - carbonarius r. flavonotatus Zik., J.F. Zikán determ.' Mischocyttarus carbonarius (Sauss.) r. flavonotatus Zik., \&, holotype, Richards, 1970'.

Current status: Mischocyttarus carbonarius (de Saussure, 1854).

\section{Mischocyttarus carinulatus Zikán, 1949: 71}

(http://hol.osu.edu/spmlnfo.html?id=MZSP\%2056955)

Holotype: ㅇ (\# MZSP 56955) '[Brazil], Esp.[írito] Santo, xi.1906, E. Garbe' 'Holotypus' 'Mischocyttarus carinulatus Zik., J.F. Zikán determ.'7.245 [Espírito Santo, E. Garbe col., Ducke rev.]"'Mischocyttarus carinulatus, $\$$, holotype'.

\section{Megacanthopus collaris Ducke, 1904: 361}

Lectotype: ㅇ (\# MZSP 61646) '[Brazil], Oyapock [now Oiapoque], 17.097"Megacanthopus collaris cotipo! Ducke rev 11' 'Dept. Zoolgia' '17.097, Oyapock' 'Mischocyttarus (Megacanthops) collaris. Lectotype Richards, 1970'.

Comments: Lectotype designated by Richards (1978: 384).

Current status: Mischocyttarus (Megacanthops) collaris (Ducke, 1904).

\section{Mischocyttarus confirmatus var. cearensis Richards, 1945: 375}

Paratype: 10' (\# MZSP 56956) 'Brazil, Estado do Ceará' 'Ceará, Serra de Baturité, 600 m, 27.7.[19]08, Ducke' 'Mischocyttartus (Meganthopus) rufidens Sauss., o', det. Ducke 1912'.

Current status: Mischocyttarus cearensis Richards, 1945.

Mischocyttarus consimilis Zikán, 1949: 148

(http://hol.osu.edu/spmlnfo.html?id=MZSP\%2056957)

Lectotype: o' (\# MZSP 56957) 'Paraguay, Mbovevo, ix.1937, Bau I, F. Schade" "o" 'Cotypus" Mischocytt. consimilis Zik., J.F. Zikán determ."Mischocyttarus consimilis Zik., o', lectotype, Richards 1970'.

Paralectotype: 19 (\# MZSP 56958) 'Paraguay, Mbovevo, ix.1937, Bau I, F. Schade, Cotypus, Mischocytt. consimilis Zik., F.J. Zikán determ.'M. consimilis Zik., , paratype'.

Comments: Lectotype designated by Richards (1978: 351).

\section{Mischocyttarus cryptobius Zikán 1935: 186}

Paralectotype: 19 (\# MZSP 56960) '[Brazil, Rio de Janeiro], Itatiaya [Itatiaia] - 1250 m km, 6 1⁄2, 10.v.1934, [?] Bau 28. J.F. Zikán"Mischocyttarus cryptobius Zik., J.F. Zikán determ."M. cryptobius Zik., ㅇ, paratype'.

Comments: Paralectotype according designation by Richards (1978: 366).

\section{Mischocyttarus cristatus Zikán, 1935: 201}

Lectotype: ㅇ (\# MZSP 61645) 'Brasil, E.[stado] do Rio [de Janeiro], Itatiaya [Itatiaia] - 700 m, 25.iii.1933, J.F. Zikán' 'Mischocyttarus cristatus Zik., J.F. Zikán determ.' 'Mischocyttarus cristatus Zik., \&, lectotype, Richards 1970'.

Paralectotypes: 1 ( \# MZSP 57094) 'Brasil, E.[stado] do Rio [de Janeiro], Itatiaya [Itatiaia] - 700 m, 25.iii.1933, J.F. Zikán"Monacanthocnemis cristatus Zik., J.F. Zikán determ.' 'M. cristatus Zik., , paratype, Richards 1970'; 10' (\# MZSP 57095) 'Brasil, [E.]stado do Rio [de Janeiro], Itatiaya [Itatiaia] - 700 m, 25.iii.1933, [?] Bau, J.F. Zikán' 'o' ‘Typus' 'Mischoccytarus cristatus Zik., J.F. Zikán determ.'M. cristatus Zik. o', paratype, Richards 1970'.

Current status: Mischocyttarus buyssoni (Ducke, 1906). 
Comments: Lectotype designated by Richards (1978: 373).

\section{Mischocyttarus crypticus Zikán, 1949: 141}

Paralectotype: 10' (\# MZSP 56959) 'Brasil, E.[stado] Rio [de Janeiro], Itatiaya [Itatiaia], 700 m, 27.v.1935, [?] Bau 59, J.F. Zikán' 'Mischocyttarus crypticus Zik., J.F. Zikán determ."M. crypticus Zik., o", paratype'.

Comments: Paralectotype according designation by Richards (1978: 366).

\section{Mischocyttarius curitybanus Zikán, 1949: 106}

Paralectotype: 19 (\# MZSP 56961) '[Brasil], S.[ão] P.[aulo], [Serra da] Cantareira, 2.436 [Serra da Cantareira, 23.iii.1906, col. H. Luderwaldt]' 'Megacanthop. cassanunga, Ducke rev. 11' 'Mischocytt. curitybanus Zik., J.F. Zikán determ.' M. curitybanus Zik.,,+ paratype'.

Comment: Paralectotype according designation by Richards (1978: 423).

\section{Mischocyttarus declaratus Zikán, 1935: 162 \\ (http://hol.osu.edu/spmInfo.html?id=MZSP\%2056962)}

Lectotype: $\sigma^{\top}$ (\# MZSP 56962) 'Brasil, E.[stado] do Rio [de Janeiro], Itatiaya [Itatiaia] - 700 m, 20.ii.1932, c.l. Bau, J.F. Zikán' 'Mischocyttarus declaratus Zik., J.F. Zikán determ' 'Mischocyttarus declaratus Zik., o', lectotype, Richards 1970 '.

Paralectotypes: 19 (\# MZSP 56963) 'Brasil, E.[stado] do Rio [de Janeiro], Itatiaya [Itatiaia] - 700 m, 6.ii.1932, [?] Bau, J.F. Zikán"Mischocyttarus declaratus Zik., J.F. Zikán determ.' 'M. declaratus Zik., ㅇ, paratype'; 1 유 (\# MZSP 56964) 'Brasil, Est.[ado] do Rio [de Janeiro], Itatiaya [Itatiaia], 700 m, 5.ii.1932, c. Bau I, J.F. Zikán"Mischocyttarus declaratus Zik., J.F. Zikán determ.'Dept. [Departament] Zoologia' 'M. declaratus Zik., o, paratype'.

Comments: Lectotype designated by Richards (1978: 348).

\section{Mischocyttarus drewseni r. andinus Zikán, 1949: 52}

Paralectotypes: 29 (\# MZSP 56965, 56966) 'Peru, Abancay, 2400 m, Dr. Weyrauch' 'Misch. drewseni r. andinus Zik., J.F. Zikán determ.' 'M. drewseni andinus Zik., ㅇ, paratype'.

Comments: Paralectotypes according designation by Richards (1978: 277).

\section{Mischocyttarus ecuadorensis Zikán, 1949: 158 (http://hol.osu.edu/spmlnfo.html?id=MZSP\%2056967)}

Holotype: ㅇ (\# MZSP 56967) 'Ecuador, 17.088 [Equador, Balzapamba, Rolle col.]' 'Mischocytt. ecuadorensis Zik., J.F.
Zikán determ."Mischocyttarus ecuadorensis Zik., Holotype, Richards 1970"'Megacanthop. rufidens, Ducke rev.11'.

\section{Mischocyttarus flaveolus Zikán, 1949: 89 \\ (http://hol.osu.edu/spmlnfo.html?id=MZSP\%2056969)}

Holotype: ㅇ (\# MZSP 56969) '[Brazil], Esp[írito] Santo, xi. 1906, E. Garbe' '7.243 [Espírito Santo, 1906, E. Garbe col.]' 'Megacanthopus surinamensis Sauss., perm. Buysson, Guyana' 'Mischocyttarus flaveolus Zik., J.F. Zikán determ. 'M. flaveolus Zik., \$, holotype = M. garbei Zik., Richards 1970'.

Current status: Mischocyttarus garbei Zikán, 1935.

\section{Mischocyttarus flavicornis Zikán, 1935: 168 (http://hol.osu.edu/spmlnfo.html?id=MZSP\%2056970)}

Lectotype: + (\# MZSP 56970) '[Brazil], Goyaz [Goiás], 17.982 [Goiás, Santa Rita de Andes, 1913]' 'Megacanth. indeterm. alffi., R.v. Ihg det. 13' 'var. alfkenii Ducke, Ducke revist.' 'Mischocyttarus flavicornis Zik., J.F. Zikán determ.' 'Mischocyttarus flavicornis Zik., ᄋ, lectotype, Richards 1970'.

Paralectotype: 19 (\# MZSP 56971) '[Brazil], Est.[ado] Goyaz [Goiás], 17.105 [Goiás, 1906, Baer col.]' 'Megac. indeterm. var. alfkenii Ducke rev. 11' Mischocytt. flavicornis Zik., J.F. Zikán determ.' 'Mischocyttarus flavicornis Zik., ㅇ, paratype'.

Comments: Lectotype designated by Richards (1978: 340).

\section{Mischocyttarus flavoscutellatus Zikán, 1935: 198} (http://hol.osu.edu/spmlnfo.html?id=MZSP\%2056972)

Lectotype: + (\# MZSP 56972) 'Brasil, E.[stado] Rio [de Janeiro], Itatiaya [Itatiaia], 700 m, 6.i.1932. J.F. Zikán' 'Mischocyttarus flavoscutellatus Zik., J.F. Zikán determ.' 'Mischocyttarus flavoscutellatus Zik., ㅇ, lectotype, Richards 1970'.

Comments: Lectotype designated by Richards (1978: 306).

\section{Mischocyttarus garbei Zikán, 1935: 194 (http://hol.osu.edu/spmlnfo.html?id=MZSP\%2056973)}

Lectotype: 9 (\# MZSP 56973) '[Brazil], Esp.[írito] Santo, Garbe leg. 06, 7.243 [Espírito Santo, com ninho, 1906, E. Garbe col.]' 'Megacanthop. surinamensis, Ducke rev. 11' 'Mischocyttarus garbei Zik., J.F. Zikán determ.' 'Mischocyttarus garbei Zik., lectotype, \&, Richards 1970'.

Comments: Lectotype designated by Richards (1978:404).

\section{Mischocyttarus gibbicolis Zikán, 1949: 43}

Paratype: 1ㅇ (\# MZSP 57103) 'Peru, Valle Chanchamayo, 800 m, 1.4[iv].1939, leg. Weyrauch' 'Mischocyttarus gibbicollis Zik., J.F. Zikán determ."'M. gibbicollis Zik., \&, paratype'. 
Current status: Mischocyttarus tomentosus Zikán, 1935.

Mischocyttarus gilvus Zikán, 1949: 159

(http://hol.osu.edu/spmlnfo.html?id=MZSP\%2056974)

Holotype: ơ (\# MZSP 56974) 'Paraguay, Pto. [Puerto] Bertoni, Coll. Bertoni' 'Megacanth. forma anormal, 16.438 [Paraguay, Puerto Bertoni, 1910, Schrottky col.], Pchrottky det. 10"Mischocytt. gilvus Zik., J.F. Zikán determ.' 'Mischocyttarus gilvus Zik., o', holotype, Richards 1970'.

\section{Mischocyytarus goyanus Zikán, 1949: 206}

(http://hol.osu.edu/spmlnfo.html?id=MZSP\%2056975)

Holotype: \& (\# MZSP 56975) '[Brazil], Est.[ado] Goyaz [Goiás], 17.085 [Goiás, 1906, Baer col.]' 'Megacanth. ater, Ducke rev. 11'Mischocytt. goyanus Zik., J.F. Zikán determ.' 'Mischocyttarus goyanus Zik., , holotype'.

Current status: Mischocyttarus imitator (Ducke, 1904).

Mischocyttarus granadaensis Zikán, 1949: 202

(http://hol.osu.edu/spmlnfo.html?id=MZSP\%2056976)

Lectotype: + (\# MZSP 56976) 'Columbia [Colombia], 17.095[Colombia, perm. Museu Paris]' 'Megacanthop. metathorac. Sauss., Ducke rev. 11' 'Mischocytt.granadensis [granadaensis] Zik., J.F. Zikán determ.' Mischocyttarus granadaensis Zik., \&, lectotype, Richards 1970'.

Current status: Mischocyttarus metathoracicus (de Saussure, 1854).

Comments: Lectotype designated by Richards (1978: 305).

\section{Mischocyttarys ignotus Zikán, 1949: 91}

Paralectotype: 19 (\# MZSP 56977) '[Brazil], E.[stado] S.[ão] P.[aulo], Franca, i.1911, E. Garbe”15.902 [São Paulo, Franca, i.1916, E. Garbe col]' 'Mischocytt. ignotus, Zik., J.F. Zikán determ.' M. ignotus Zik., \&, paratype'.

Comments: Paralectotype according designation by Richards (1978: 403).

\section{Mischocyttarus inca Zikán, 1949: 191}

(http://hol.osu.edu/spmlnfo.html?id=MZSP\%2056978)

Holotype: \& (\# MZSP 56978) 'Perú, 1.193 [Peru, Stand col.], Mischocyttarus (Megacanthopus) rufidens Sauss det. Ducke 1912' 'Mischoc. bequaerti r. inca Zik., J.F. Zikán determ.'Mischocyttarus silvaticus Zik. race inca Zik. 1949, o, holotype, Richards, 1970'.

\section{Mischocyttarus infrastrigatus Zikán 1949: 175}

Paralectotypes: 19 (\# MZSP 56979) 'Brasil, E.[stado] do Rio [de Janeiro], Itatiaya [Itatiaia], 700, 11.ii.1941, Bau 113, J.F. Zikán' ‘Mischocytt. ornatulus Zik., J.F. Zikán de- term.' 'M. ornatulus Zik., 9, paratype'; $10^{7}$ (\# MZSP 56980) 'Brasil, E.[stado] do Rio [de Janeiro], Itatiaya [Itatiaia], 700 m, 1.iv.1941, Bau 114, J.F. Zikán"Mischocytt. ornatulus Zik., J.F. Zikán determ.' M. ornatulus Zik., o', paratype'.

Comments: Paralectotypes according designation by Richards (1978: 360).

\section{Mischocyttarus intermedius Zikán, 1949: 57}

Paralectotype: 19 (\# MZSP 57093) 'Brasil, E.[stado] do Rio [de Janeiro], Itatiaya [Itatiaia] - 700 m, 26.iii.1933, [?] Bau, J.F. Zikán' 'Mischoc. intermedius Zik., J.F. Zikán determ.' M. intermedius Zik.,, , paratype'.

Current status: Mischocyttarus drewseni de Saussure, 1857.

Comment: Paralectotype according designation by Richards (1978: 276).

\section{Mischocyttarus itatiayaensis Zikán, 1935: 190}

Paralectotype: 19 (\# MZSP 56981) '[Brazil, Rio de Janeiro], Itatiaya [Itatiaia], 900 m km, Itaoca, 5.xi.1931, [?] Bau, J.F. Zikán' Mischocyttarus itatiayaensis Zik., J.F. Zikán determ., Mischcyttarus itatiayaensis Zik., \$, paratype'.

Comments: Paralectotype according designation by Richards (1978: 353).

\section{Mischocyttarus japuraensis Zikán, 1949: 147}

(http://hol.osu.edu/spmlnfo.html?id=MZSP\%2056982)

Holotype: ㅇ (\# MZSP 56982) '[Brazil], Est.[ado] Amaz. [onas], Rio Japurá, 17.106 [Amazonas, Rio Japurá, ix.04, Ducke col.]' 'Megac. indet. var. alfkenii, Ducke rev.11' 'Mischocytt. japuraensis Zik., J.F. Zikán determ.'

Current status: Mischocyttarus alfkenii alfkenii (Ducke, 1904).

Mischocyttarus (Kappa) juruanus Richards, 1978: 302 (http://hol.osu.edu/spmlnfo.html?id=MZSP\%2056983)

Holotype: \& (\# MZSP 56983) '[Brazil], E.[stado] Amaz[onas], Rio Juruá, 1254 [Amazonas, Rio Juruá, com ninho, 1902, E. Garbe col.]' 'Megacanthop. injucundus Sauss., Ducke rev. 11' 'Dept. [Departament] Zoologia' 'Mischocyttarus juruanus Rich., + , holotype'.

Current status: Mischocyttarus injucundus (de Saussure, 1854).

Mischocyttarus lanei Zikán, 1949: 127

(http://hol.osu.edu/spmlnfo.html?id=MZSP\%2056997)

Holotype: 9 (\# MZSP 56997) '[Brazil], São Paulo, Campos da Serra, xi.[19]40"Mischocytt. lanei Zik., J.F. Zikán determ.' 'Mischocyttarus lanei Zik., \$, Holotype, Richards 1970'. 
Comments: In the original publication the collection date was incorrectly transcribed as 1904.

\section{Mischocyttarus litoralis Zikán, 1949: 53}

Paralectotype: 1 ( \# MZSP 57092) 'Brasil, Rio de Janeiro - 30 m., Deodoro, Lab.[oratório] de Pomologia, 16.viii. [1]935, W. Zikán' 'Misch. intermedius r. litoralis Zik., J.F. Zikán determ.' 'M. litoralis Zik., probably $\$$ paratype, Richards 1970'.

Current status: Mischocyttarus drewseni de Saussure, 1857.

Comments: Paralectotype according designation by Richards (1978: 276).

\section{Mischocyttarus litoralis r. meridionalis Zikán, 1949: 54 \\ (http://hol.osu.edu/spmlnfo.html?id=MZSP\%2056968)}

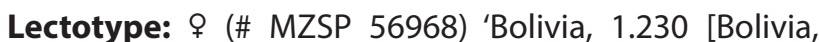
Ducke rev. 11]' 'Mischocyth. drewseni Sauss., Ducke, rev. 11' 'Mischoc. drewseni r. meridionalis Zik., J.F. Zikán determ.' Mischocyttarus litoralis Zik. race meridionalis Zik. + , lectotype, Richards, 1970'.

Current status: Mischocyttarus drewseni andinus Zikán, 1949.

Comments: Lectotype designated by Richards (1978: 277).

Mischocyttarus mattogrossensis Zikán, 1935: 156

(http://hol.osu.edu/spmlnfo.html?id=MZSP\%2056985)

Holotype: ơ (\# MZSP 56985) '[Brazil], Estado M.[ato] Grosso, 3.147[Mato Grosso, 1904, E. Garbe col., Ducke rev.11]' 'Mischocyth. labiatus F. Ducke rev. 11' 'Mischocyttarus mattogrossensis, Zik., J.F. Zikán determ.' 'Mischocyttarus mattogrossensis Zik., o', holotype'.

\section{Mischocyttarus montei Zikán, 1949: 65}

Paralectotypes: 20' (\# MZSP 56986, 56987) '[Brazil], (Est. [ado] S.[ão] P.[aulo]), 15.782 [São Paulo, Franca, x.1910, E. Garbe col.]' 'Mischocyttarus (Megacanthopus) lecointei Ducke, det.Ducke, 1912"Vespidae,Megacanthopuslecointei Ducke, Garbe - Franca, Est.[ado] S.[ão] P.[aulo], 15.782 [São Paulo, Franca, x.1910, E. Garbe col.]"Mischocyttarus montei Zik., J.F. Zikán determ.'M. montei Zik, o’, paratype'.

Comments: In the original publication the museum number was incorrectly transcribed as 15.786. Paralectotypes according designation by Richards (1978: 377).

\section{Mischocyttarus nigroclavatus Zikán, 1949: 92}

Paratype: 10' (\# MZSP 56988) 'Costa Rica, S.[an] José, 25.i.1926, F. Nevermann [col], Bau 5' 'Mischocytt. nigro- clavatus Zik., J.F. Zikán determ.' 'M. nigroclavatus Zik., o", paratype'.

Comments: In the original publication the collection date was incorrectly transcribed as 1936.

\section{Mischocyttarus oaxacanus Zikán, 1949: 168}

Syntypes: 10' (\# MZSP 56989) '16.476 [Mexico, 1912], Buysson det.'Museum Paris, Basse Mixtéque, Env. d'Oaxaca, L. Diguet, 1904' 'Mischoc. pallidipectus Sm. r. oaxacanus Zik., J.F. Zikán determ.' 'Mischocyttarus oaxacanus Zik., o' lectotype, Richards 1970'; 19 (\# MZSP 56990) same data except 'Mischocyttarus oaxacanus Zik., $\$$ paratype, Richards 1970'; 1 ( \# MZSP 56991) 'Mexique, State de Puebla, Env. De Zehuacan' 'Mexico, 1.831 [Mexico, Puebla, Ducke rev.11]' 'Meganthop. rufipens, Ducke rev. 11' 'Mischoc. pallidipectus Sm. r. oaxacanus Zik., J.F. Zikán determ.' M. oaxacanus, Zik., + , paratype'.

Current status: Mischocyttarus pallidipectus (Smith, 1857).

\section{Mischocyttarus pallidus Zikán, 1949: 116 \\ (http://hol.osu.edu/spmlnfo.html?id=MZSP\%2056998)}

Holotype: 9 (\# MZSP 56998) ‘[Brazil], Est.[ado] Amazonas, 1.067 [Manaus, Bicego leg., Ducke rev. 11]"Megacanthop. surinamensis Ducke rev. 11' 'Mischocytt. pallidus Zik., J.F. Zikán determ.'Mischocyttarus pallidus Zik., ㅇ, Holotype'.

\section{Mischocyttarus paraguayensis Zikán, 1935: 165 (http://hol.osu.edu/spmlnfo.html?id=MZSP\%2056992)}

Lectotype: o' (\# MZSP 56992) 'Paraguay, Mbovevo, 25.iii.1933, F. Schade, [?] Bau' 'Mischocyttarus paraguayensis Zik., J.F. Zikán determ.' 'Mischocyttarus paraguayensis Zik., ơ', lectotype, Richards 1970'.

Comments: In the original publication the collection date was incorrectly transcribed as 1932. Lectotype designated by Richards (1978: 339).

\section{Mischocyttarus parallelogrammus Zikán, 1935: 172}

Lectotype: 9 (\# MZSP 61644) 'Brasil, E.[stado] do Rio [de Janeiro], Itatiaya [Itatiaia], 700 m, 6.vi.1932, Monte Bello. Bau 2, J.F. Zikán' 'Mischocyttarus parallelogrammus Zik., J.F. Zikán determ.' 'O' 'Typus' 'Mischocyttarus parallelogrammus Zik. Lectotype, Richards, 1970'.

Paralectotype: 19 (\# MZSP 57094) '[Brazil], Est.[ado] do Rio [de Janeiro], Itatiaya [Itatiaia], 700 m, 6.vi.1932, Monte Bello. J.F. Zikán"Mischocytt. parallelogrammus Zik., J.F. Zikán determ.'M. parallelogrammus Zik, \$, paratype'.

Mischocyttarus pedunculatus Zikán, 1935: 159 (http://hol.osu.edu/spmlnfo.html?id=MZSP\%2056993)

Lectotype: + (\# MZSP 56993) '[Brazil, Rio de Janeiro], Itatiaya [Itatiaia] - 550 m. km, 3.ii.1933, Faz.[enda] Val 
Paraiso, [?] Bau 2' 'Mischocyttarus pedunculatus Zik., J.F. Zikán determ.'Mischocyttarus pedunculatus Zik., ㅇ, lectotype, Richards 1970'.

Paralectotypes: 19 (\# MZSP 56994) 'Brasil, E.[stado] do Rio [de Janeiro], Itatiaya [Itatiaia] - 700 m, 17.ii.1933, Bau M. Bello, J.F. Zikán, Mischocyttarus pedunculatus Zik., J.F. Zikán determ.'Mischocyttarus pedunculatus Zik., J.F. Zikán determ!

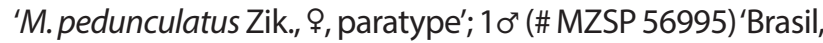
E.[stado] do Rio [de Janeiro], Itatiaya [Itatiaia] - 700 m, 3.iii.1933, J.F. Zikán' 'Mischocyttarus pedunculatus Zik, J.F. Zikán determ."Mischocyttarus pedunculatus Zik, ơ', paratype'.

Current status: Mischocyttarus wagneri (du Buysson, 1908).

Comments: Lectotype designated by Richards (1978: 334).

\section{Mischocyttarus pelor Carpenter, 1988: 89 in Carpenter \& Wenzel, 1988}

Paratype: 19 (\# MZSP 56996) 'Costa Rica, Turrialba, 31.May.1951, OLCartwright' 'Paratype, Mischocyttarus pelor Carpenter'.

\section{Mischocyttarus proximus Zikán, 1949: 130}

(http://hol.osu.edu/spmlnfo.html?id=MZSP\%2056984)

Holotype: 9 (\# MZSP 56984) ‘[Brazil], São Paulo, Campos da Serra, xi.40' 'Mischocytt. proximus Zik., J.F. Zikán determ.'Mischocyttarus proximus Zik., o, holotype, Richards 1970'.

\section{Mischocyttarus riograndensis Richards, 1978: 361 (http://hol.osu.edu/spmlnfo.html?id=MZSP\%2056999)}

Holotype: 9 (\# MZSP 56999) '[Brazil], Rio Grande do Sul, S.[ão] Leopoldo, Brentano col, viii.[19]13, 18.490 [Rio Grande do Sul, São Leopoldo, viii.1913, Pe. Brentano col.]' 'Megacanthop. indeterminabilis Sauss., R.v. Iherig det.' 'Mischocyth. surinamensis Sauss., Ducke rev. 1911' 'Mischocytt. riograndensis Zik., J.F. Zikán determ.' 'Mischocyttarus riograndensis Rich., \&, holotype'.

Paratypes: 29 (\# MZSP 57000, 57001) '18.490 [Rio Grande do Sul, São Leopoldo, viii.1913, Pe. Brentano col.]' 'Mischocytt. riograndensis Zik., J.F. Zikán determ.' 'Mischocyttarus riograndensis, + , Rich., paratype'; 10' (\# MZSP 57002) '[Brazil], Rio Grande do Sul, 1.210 [Rio Grande do Sul]' 'Megacanthop. indeterminab. Ducke rev. 11 ' 'Mischocytt. riograndensis Zik., J.F. Zikán determ.' 'Mischocyttarus riograndensis, o', Rich, paratype'.

\section{Mischocyttarus santacruzi Raw, 2000: 941}

(http://hol.osu.edu/spmlnfo.html?id=MZSP\%2057003)

Holotype: 9 (\# MZSP 57003) 'Brazil, BA[Bahia], Ilhéus, Faz. [enda] Baixão, 12.2.1999, A. Raw' 'Mischocyttarus santacruzi Raw'.
Paratype: 10' (\# MZSP 57004) 'Brazil, BA[Bahia], Ilhéus, Faz.[enda] Baixão, 12.2.1999, A. Raw"'Mischocyttarus santacruzi Raw 2000'.

\section{Mischocyttarus saussurei Zikán, 1949: 98}

Paratype: 10' (\# MZSP 57005) '[Brazil], São Paulo, Ypiranga [Ipiranga], anno [ano] 1922' 'Mischocytt. saussurei Zik., J.F. Zikán determ.' Mischocyttarus saussurei Zik., $\sigma^{\prime \prime}$, paratype'.

\section{Mischocyttarus (Clypeopolybia) sericeus Richards, 1978: 283}

(http://hol.osu.edu/spmlnfo.html?id=MZSP\%2057006)

Holotype: 9 (\# MZSP 57006) 'Bolívia, Yungas de La Paz, 16.979 [Bolivia, Yungas de La Paz, 1000, camp. Rolle col.]' 'Polybia sericea, Ducke rev. 12' 'Mischocyttarus sericeus Rich., \&, Holotype'.

\section{Mischocyttarus schrottkyi Zikán, 1949: 159} (http://hol.osu.edu/spmlnfo.html?id=MZSP\%2057007)

Holotype: 9 (\# MZSP 57007) ‘Paraguay, P.[uerto] Bertoni, Coll.[ection] Bertoni' 'Paraguay, 16.438 [Paraguay, Puerto Bertoni, 1910, Schottky col.], Schrottkyi det.' Mischocytt. schrottkyi Zik., J.F. Zikán determ.' Mischocyttarus schrottkyi Zik., , , holotype, Richards 1970'.

Current status: Mischocyttarus consimilis Zikán, 1949.

Mischocyttarus tomentosus Zikán, 1935: 155

(http://hol.osu.edu/spmlnfo.html?id=MZSP\%2057008)

Holotype: 9 (\# MZSP 57008) '[Brazil], Esp.[írito] Santo, 7.244 [Espírito Santo, 1906, E. Garbe col.], Garbe leg. 06' 'Mischocyth. Iabiatus F., Ducke rev. 11' Mischocyttarus tomentosus Zik., J.F. Zikán determ."'Mischocyttarus tomentosus Zik., ㅇ, holotype, Richards 1970'.

\section{Mischocyttarus varolii Zikán, 1949: 95}

Paratype: 19 (\# MZSP 57009) '[Brazil], S.[ão] P.[aulo], Franca, 10.xii.1913, E. Garbe'‘17.499 [São Paulo, Franca, xii.1910, E. Garbe col.]' 'Mischocyttarus varolii Zik., J.F. Zikán determ.' 'M. varolii Zik., \$, paratype = cerbereus Ducke ssp. styx, Rich. 1970'.

Current status: Mischocyttarus cerberus styx Richards, 1940.

\section{Mischocyttarus ypiranguensis Fonseca, 1926: 181} (http://hol.osu.edu/spmlnfo.html?id=MZSP\%2057010)

Lectotype: o' (\# MZSP 57010) '[Brazil], São Paulo, Ypiranga [Ipiranga, neighborhood], 19. 287 [Ipiranga, ii.1916]' 'Mischocyttarus ypiranguensis, Typo, Pinto F. 23' 'Mischocyttarus ypiranguensis Pinto da Fonseca, lectotype, Richards 1970'. 
Paralectotypes: $30^{\prime \prime}$ and 29 (\# MZSP 57011-57015) '[Brazil], São Paulo, Ypiranga [Ipiranga, neighborhood], 19. 287 [Ipiranga, ii.1916]' 'Mischocyttarus ypiranguensis, Typo, Pinto F. 23' 'Miscchocyttarus ypiranguensis Pinto da Fonseca, paratype, Richards 1970'.

Comments: Lectotype designated by Richards (1978: 427).

Mischocyttarus (Kappa) zikaninus Richards, 1978: 295 (http://hol.osu.edu/spmlnfo.html?id=MZSP\%2057016)

Holotype: \& (\# MZSP 57016) 'Brazil, Estado do Pará' 'Óbidos, 30.5.1911, Ducke' 'Mischocyttarus (Megacanthopus) pseudomimeticus Schulz, det. Ducke 1912"Mischocyttarus zikaninus Rich., holotype'.

\section{Polistes Latreille}

Polistes brevifissus Richards, 1978: 481

(http://hol.osu.edu/spmlnfo.html?id=MZSP\%2057043)

Holotype: 9 (\# MZSP 57043) '[Brazil], MT [Mato Grosso], Utiariti, Rio Papagaio, 17.x.1966, Lenko \& Pereira' 'Polistes brevifissus, Rich., $\$$, holotype'.

Polistes canadensis borientalis Richards, 1978: 494 (http://hol.osu.edu/spmlnfo.html?id=MZSP\%2057022)

Holotype: 9 (\# MZSP 57022) 'Brasil, Pernambuco, Serra Talhada, 7.v.1965, G. Cisneiros col.' 'Polistes canadensis (L.) ssp. borientalis Rich., $\$$, holotype'.

Paratypes: 2 9 (\# MZSP 57023, 57024) '[Brazil], Paraíba, Corema, vi.1957, Exp.[edição] Dep.[artamento] Zoologia' 'Polistes canadensis (L.) ssp. borientalis Rich., \&, paratype'; 129 (\# MZSP 57025-57036) 'Brasil, Pernambuco, Serra Talhada, 7.v.1965, G. Cisneiros col.' 'Polistes canadensis (L.) ssp. borientalis Rich., ㅇ, paratype'; 5 ㅇ (\# MZSP 57037-57041) '[Brazil], R.[io] G.[rande] [do] Norte, Natal, vii.[1]955, Pe. Pereira' 'P. canadensis (L.) ssp. borientalis Rich., ㅇ, paratype'; 1 우 (\# MZSP 57042) '[Brazil], R.[io] G.[rande] [do] Norte, Natal, viii.x.[1]954' 'P. canadensis

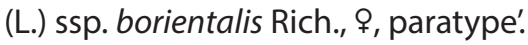

Comments: Original publication reported 15 females paratypes from Serra Talhada (Pernambuco, Brazil) deposited at MZSP, however, three paratypes were not found.

\section{Polistes bicolor var. unicolor Ducke, 1908: 700}

(http://hol.osu.edu/spmlnfo.html?id=MZSP\%2057021)

Lectotype: ㅇ (\# MZSP 57021) '[Panama], Chiriqui, de Standing, 1.156 [Chiriqui, Stand. col.]' Polistes bicolor var. unicolor Ducke, Ducke det. 11' '98358' 'P. bicolor var. unicolor Ducke, + , lectotype, Richards 1971' 'Polistes duckei Bequaert, Type'.
Current status: Polistes bicolor Lepeletier, 1836.

Comments: Lectotype designated by Richards (1978: 531).

\section{Polistes notatipes Richards, 1978: 488}

(http://hol.osu.edu/spmlnfo.html?id=MZSP\%2057018)

Holotype: 9 (\# MZSP 57018) ‘[Brazil, São Paulo], Ypiranga [lpiranga, neighborhood], 647 [Ipiranga, 6.ii.1902 J Lima col.]'97577"'Polistes notatipes Rich., , holotype'.

Paratypes: 19 (\# MZSP 57019) 'Brasil, Rio Grande do Sul, Ihering' '97603' 'Polistes notatipes Rich., \&, paratype'; 1 \% (\# MZSP 57020) 'Brasil, RS [Rio Grande do Sul], 1888, R. v. Ihering, almost certainly' '97598' 'Polistes notatipes Rich., \$, paratype'.

Comments: In addition to the specimens listed, the original publication reported more two females paratypes from Rio Grande do Sul (Brazil) and one female from São Paulo (Brazil) as deposited at MZSP, however, these paratypes were not found in the colletion.

\section{Zethinae \\ Zethus Fabricius}

\section{Zethus (Zethusculus) brasiliensis fuscatus Bohart \& Stange, 1965: 130}

Paratype: 19 (\# MZSP 57078) '[Brazil], Amazon [Amazonas state], Rio Uapes' '10.779 [Brazil, Amazonas, Rio Uaupés, 1907, Dr Bosh col.]' 'Zethus coeruleopennis Fabri, C. Schrottky det. 1910' '01725' 'Lane' 'Paratype Zethus brasiliensis fuscatus Bohart \& Stange'.

\section{Discoelius bruneoniger Ihering, 1911: 469 \\ (http://hol.osu.edu/spmlnfo.html?id=MZSP\%2057083)}

Holotype: + (\# MZSP 57083) '[Brazil], Est.[ado] Goyaz [Goiás], G.A. Baer Leg, Discoelius bruneoniger n. sp., typus R. v Ihering, 6.803' '6.803 [Brazil, Goiás, 1906, Baer coll.]' '97983' 'Lane' 'Zethus bruneoniger Ihering, Type' 'Zethus (Zethus) productus Fox det R.M. Bohart \& L.A. Stange, [19]63'.

Current status: Zethus (Zethus) productus (Fox, 1899).

Discoelius peculiaris dives Ihering, 1911: 471 (http://hol.osu.edu/spmlnfo.html?id=MZSP\%2057082)

Holotype: 9 (\# MZSP 570782) 'Perú? Bolivia?, 16.333 Didymogaster peculiaris dives n. sp., Typus R. v. Ihering' '16.333 [Peru? Bolivia?]' 'Lane' '97979' 'Perú? Bolívia?, Didy.[mogaster] peculiaris dives, Ihering, Type' 'Holotype, Discoelius peculiaris dives $\$$, Ihering' 'Zethus (Zethus) fuscus \% (Perty), det R.M. Bohart \& L.A. Stange, 1[9]63'.

Current status: Zethus (Zethus) fuscus (Ihering, 1911). 


\section{Discoelius luederwaldti lhering, 1911: 468}

(http://hol.osu.edu/spmlnfo.html?id=MZSP\%2057080)

Holotype: ơ (\# MZSP 57080) '[Brazil, São Paulo, São Paulo], Ypiranga [Ipiranga, neighborhood], [Luederwaldt coll.] typo' '3.389 [Brazil, São Paulo, São Paulo, Ipiranga, 20vii1906]"97997"Lane' Holotype Discoelius luederwaldti o' Ihering' 'Discoelius heydeni o' (Sauss) C. Schrottky det. 1910'3.389 Discoelius luederwaldti n. sp., Typus, R. v. Ih., H. Luederwaldt leg. Ypiranga'.

Current status: Zethus (Zethoides) luederwaldti (Ihering, 1911).

\section{Zethus (Zethus) pronatus Bohart \& Stange, 1965: 60} (http://hol.osu.edu/spmlnfo.html?id=MZSP\%2057078)

Holotype: 9 (\# MZSP 57078) ‘[Brazil, São Paulo] Camp.[os] d.[o] Jordão Est. S.P.'16.330 [Brazil, São Paulo, Campos do Jordão, xii.1905, Luderwaldt coll.]' 'Discoelius fluminensis Brèthes, C. Schrottky det. 1910' '97996' 'Lane' 'Holotype Zethus pronatus + Bohart \& Stange'.

\section{Zethus (Zethusculus) mexicanus erythrogena Bohart \& Stange, 1965: 132}

Paratypes: 10' (\# MZSP 57081) '[Brazil] São Paulo, Jundiahy [Jundiaí], iii.[19]08, 14.862"Zethus mexicanus, C. Schrottky det. 1910"'101713'Lane'"Paratype Zethus mexicanus erythrogena, Bohart \& Stange'; 19 '[Brazil] Minas Geraes [Minas Gerais], Pirapora, 17.417 [Brasil, Minas Gerais, Pirapora, 1912, Garbe col.]' 'Zethus mexicanus v. brasiliensis, Bequaert det. 1930'‘101721' 'Lane' 'Paratype Zethus mexicanus erythrogena, ㅇ, Bohart \& Stange'.

Comments: In addition to the specimens listed, the original publication reported one female from Jundiaí (São Paulo, Brazil) and one male from Pirapora (Minas Gerais, Brazil) deposited at MZSP, however, these paratypes were not found in the colletion.

\section{Zethus (Zethusculus) schrottkyanus Ihering, 1911: 465 (http://hol.osu.edu/spmlnfo.html?id=MZSP\%2057084)}

Lectotype: $\sigma^{\top}$ (\# MZSP 57084) '[Brazil], Rio Gr.[ande] do Sul, [Camaquã], H.v.lh[ering] leg, Zethus schrottkyanus $\sigma^{7}$ n. sp., typus, R.v.lh, 1514"'1514 [Brazil, Rio Grande do Sul, Ihering coll.]"101726"Lane"Z. (Zethusculus) schrottkyanus Ihering, Lectotype, Rio Gr. do Sul' Z. (Zethusculus) schrottkyanus $\sigma^{7}$ (Ihering) det. R.M. Bohart \& L.A. Stange [19]63' 'Lectotype Zethus schrottkyanus Iher. Bohart \& Stange'.

Comments: Lectotype designated by Bohart \& Stange (1965: 148).

\section{ACKNOWLEDGEMENTS}

This work was supported by Programa de Capacitação em Taxonomia - PROTAX grant to CRFB with fund of
Conselho Nacional de Desenvolvimento Científico e Tecnológico (CNPq 440574/2015-3) and Fundação de Amparo à pesquisa do Estado de São Paulo (FAPESP 2016/50378-8). TOA, KSR, and ADS are also grateful to PROTAX/CNPQ for the fellows (proc. 152579/2016-8, 150409/2016-8, and 134124/2016-2, respectively). HCO thanks to Fundação Coordenação de Aperfeiçoamento de Pessoal de Nível Superior (CAPES 88887.136354/2017-00) for post-doctoral grants. We also thanks to Mônica Ulysséa for support funding articles and scanning old manuscript catalogs of insect specimens deposited in the MZUSP, Cecilia Wichert for the help with informations about Pompilidae, Marcel Hermes and Bolivar Garcete-Barrett for the clarifications about species of Hypalastoroides, Lívia Pires do Prado for provide data of loaned specimens, Dr. Norman F. Johnson and Sara Hemly for helping with the upload of photos to Hymenoptera Online database.

\section{REFERENCES}

Andena, S.R. \& Carpenter, J.M. 2011. A new species of Metapolybia (Hymenoptera: Vespidae; Polistinae, Epiponini). Entomologica Americana, 117: 117-120. D0I

Bohart, R.M. \& Stange, L.A. 1965. A revision of the genus Zethus Fabricius in the western hemisphere (Hymenoptera: Eumenidae). University of California Publications in Entomology, 40: 1-208.

Bradley, J.C. 1911. A new Thynnid Wasp from Brazil (Hym.) Deutsche Entomologische Zeitschrift, 131-132.

Brandão, C.R.F. 1991. Adendos ao catálogo abreviado das formigas da região Neotropical (Hymenoptera: Formicidae). Revista Brasileira de Entomologia, 35: 319-412.

Brandão, C.R.F.; Esteves, F.A. \& Prado, L.P. 2010. A Catalogue of the Pseudomyrmecinae ants type specimens (Hymenoptera, Formicidae) deposited in the Museu de Zoologia da Universidade de São Paulo, Brazil. Papéis Avulsos de Zoologia, 50: 693-699. D01

Brèthes, J. 1908. Contribuición preliminar para el conocimiento de los Pepsis. Anales del Museo de História Natural de Buenos Aires, 10: 233-243.

Brèthes, J. 1911. Quelques nouveaux Céropalides (Hymenopt.) du Musée de São Paulo. Revista do Museu Paulista, 8: 64-70.

Brèthes, J. 1914. Contribuition à l'êtude des Pepsis. Anales del Museo de História Natural de Buenos Aires, 26: 235-360.

Burmeister, H. 1872. Ueber die Pompiliden und Sphegiden des La Plata Gebietes. Stettiner Entomologische Zeitung, 33: 230-241.

Buysson, R.D. 1908. Hyménoptères nouveaux. Revue Entomologique, 27: 207-219.

Carpenter, J.M. 2018. Checklist and/or Catalog of Social Wasps. Natural History Laboratory, Ibaraki University and World Association for the Study of Paper Wasps Japan. Avaiable at: http://iunh2.sci.ibaraki.ac.jp/ wasp/list.html. Access in: 01/03/2018.

Carpenter, J.M. \& Wenzel, J.W. 1988. A new species and nest type of Mischocyttarus from Costa Rica (Hymenoptera: Vespidae; Polistinae), with descriptions of nests of three related species. Psyche, 95: 89-99. D0I

Casal, 0.H. 1957. Nuevas especies de Mutillidae (Hymenoptera). Neotropica, 3: $90-69$.

Casal, 0.H. 1961. Mutillidae neotropicales VI. Notas del Museo de la Plata, 20: 57-61.

Casal, 0.H. 1962a. Mutillidae neotropicales XV (Hymenoptera). Tres especies nuevas del género Tallium André. Revista Brasileira de Biologia, 22: 317-326. 
Casal, 0.H. 1962b. Mutillidae neotropicales V (Hymenoptera). Espécies nuevas de la Argentina del género Tallium. Acta Zoologica Lilloana, 18: 263-279.

Casal, 0.H. 1963. Mutillidae neotropicales XXI (Hymentoptera). Comentários sobre Atillum André com la descripción de espécies nuevas y el alotipo de A. stygium Mickel. Revista de la Sociedad Entomológica Argentina, 24: 89-91.

Casal, 0.H. 1969. Sobre Traumatomutilla André (Hymenoptera, Mutillidae). Physis, 28: 279-298.

da Fonseca, P. 1926. Uma nova espécie de vespa social do gênero Mischocyttarus. Revista do Museu Paulista, 14: 179-187.

Dahlbom, A.G. 1843. Hymenoptera Europaea praecipue borealia; formis typicis nonnulis Specierum Generumve Exoticorum aut Extraneorum propter nexum systematicus associates; per Familias, Genera, Species et Varietates desposita atque descripta. Tomus: Sphex in sensu Linneano. Lund. Officina Lundbergiana, 1: 1-172.

Dalla Torre, C.G. DE. 1897. Catalogues Hymenopteroum hucusque descriptorum systematicus et synonymicus 8. Fossores, I-IX: 1-750.

Ducke, A. 1904. Sobre as vespidas sociaes do Pará. Boletim do Museu Paraense Emilio Goeldi, 4: 317-374.

Ducke, A. 1907a. Contribuition à la connaissance des Scoliides de I'Amérique du Sud. Revue d'entomologie, 26: 5-9.

Ducke, A. 1907b. Novas contribuições para o conhecimento das vespas (Vespidae Sociales) da região neotropical. Boletim do Museu Paraense Emilio Goeldi, 5: 152-199.

Ducke, A. 1908. Beitrãge zur Hymenopterenkunde Amerikas. Deutsche Entomologische Zeitschrift, 695-700.

Ducke, A. 1910. Révision des guêpes sociales polygames d'Amérique. Annales Musei historico-naturalis hungarici, 8: 449-544.

Ducke, A. 1914. 0 género Pterombrus Sm. (Hymen.). Revista do Museu Paulista, 9: 107-122.

Esteves, F.A.; Brandão, C.R.F. \& Prado, L.P. 2011. The type specimens of "Dorylomorph" ants (Hymenoptera, Formicidae: Aenictinae, Ecitoninae, (erapachynae, Leptanilloidinae) deposited in the Museu de Zoologia da Universidade de São Paulo, Brazil. Papéis Avulsos de Zoologia, 51: 341-357. D01

Fox, W.J. 1899. Contribuitions to a knowledge of the Hymenoptera of Brazil, no. 6 - a collection from Rio Grande do Sul and São Paulo. Proceedings of the Academy of Natural Sciences of Philadelphia, 51: 195-200.

Genise, J. \& Kimsey, L. 1991. New Genera of South America Thynninae (Tiphiidae, Hymenoptera). Psyche, 98: 57-69. DOI

Genise, J.F. \& Kimsey, L.S. 1993. Revision of the South American Thynnine genus Elaphroptera Guérin-Méneville (Hymenoptera: Tiphiidae). Journal of Hymenoptera Research, 2: 195-220. DOI

Gerstaecker, A. 1874. Mutillarum Americae meridionalis indigenarum synopsis systematica et synonymica. Archiv für Naturgeschichte, 40: 41-77.

Hymenoptera Online (HOL). 2016. Avaiable at: http://hol.osu.edu. Access in: 01/03/2018.

Ihering, H. von. 1896. L'êtat des guêpes sociales du Brésil. Bulletin de la Société Zoologique de France, 21: 159-162.

Ihering, H. von. 1903. Contribuitions á l'etude des vespides de I'Amérique du Sud. Annales de la Société Entomologique de France, 72: 144-155.

Ihering, H. von. 1904. As vespas sociaes do Brasil. Revista do Museu Paulista, 6: 97-309.

Ihering, R. 1911. Algumas espécies novas de vespas solitárias (Eumenidae). Revista do Museu Paulista, 8: 462-475.

International Comission on Zoological Nomenclature (ICZN). 1999. International Code os Zoological Nomenclature. $4^{\mathrm{a}}$ ed., London. Available at: www.nhm.ac.uk/hosted-sites/iczn/code. Access in: 17/01/2018.

Integrated Taxonomic Information System (ITIS). 2018. Avaiable at: www.itis. usda.gov. Access in: 01/03/2018.
Kawada, R. \& Buffington, M.L. 2016. A scalabe and modular dome illumination system for scientific microphotography on a budget, PLOS ONE, Article ID e0153426. D0I

Kimsey, L.S. 1991a. Additional new genera and species of South American thynnine wasps (Hymenoptera: Tiphiidae). Psyche, 98: 71-80. DOI

Kimsey, L.S. 1991b. Revision of the South American wasp genus Aelurus (Hymenoptera: Tiphiidae: Thynninae). Systematic Entomology, 16: 223-237. DOI

Kimsey, L.S. 1996. Revision of the South American Thynninae genus Upa (Hymenoptera: Tiphiidae). Proceedings of the Entomological Society of Washington, 98: 55-63.

Kimsey, L.S. 2005. Revision of the Northern South American tiphiid genus Merithynnus Kimsey, 1991 (Hymenoptera: Tiphiidae: Tiphiinae). Proceedings of the Entomological Society of Washington, 107: 576-595. DOI

Klingenberg, C. \& Brandão, C.R.F. 2005. The type specimens of fungus growing ants, Attini (Hymenoptera, Formicidae, Myrmicinae) deposited in the Museu de Zoologia da Universidade de São Paulo, Brazil. Papéis Avulsos de Zoologia, 45: 41-50. DOI

Mickel, C.E. 1937. New World Mutillidae in the Spinola collection at Torino, Italy (Hymenoptera). Rio de Janeiro, Brasil. Revista de Entomologia, 7: 165-207.

Mickel, C.E. 1938. The Neotropical mutillid wasps of the genus Timulla Ashmead (Hymenoptera: Mutillidae). Transactions of the Royal Entomological Society of London, 87: 529-680. D01

Mickel, C.E. 1939a. A monograph of the Neotropical mutillid genus Hoplomutilla Ashmead (Hymenoptera: Mutillidae). Revista de Entomologia, 10: 337-403.

Mickel, C.E. 1939b. A monograph of the Neotropical mutillid genus Hoplomutilla Ashmead (Hymenoptera: Mutillidae) (conclusion). Revista de Entomologia, 10: 641-717.

Mickel, C.E. 1941. Monograph of the South American mutillid genus Hoplocrates Mickel (Hymenoptera: Mutillidae). Revista de Entomologia, 12: 341-414.

Mickel, C.E. 1960. A review of the mutillid genus Cephalomutilla André (Mutillidae: Hymenoptera). Revista Brasileira de Entomologia, 9: 157-168.

Naumann, M.G. 1968. A revision of the genus Brachygastra (Hymenoptera: Vespidae). University of Kansas Science Bulletin, 17: 929-1003.

Oliveira, M.L.; Fernandez, D.R.R.; Alvarenga, T.M.; Andena, S.R.; Araujo, R.O.; Azevedo, C.0.; Barbosa, D.N.; Bartholomay, P.R.; Costa, V.A.; DalMolin, A.; Fernandes, I.0.; Gadelha, S.S.; Galhardo, F.; Hermes, M.G.; Justino, C.E.L; Kawada, R.; Kohler, A.; Lara, R.I.R.; Lucena, D.A.A.; Luz, D.R.; Macedo, A., Maragaría, C.; Oliveira, B.G.; Pádua, D.G.; Perioto, N.W.; Pikart, T.G.; Querino, R.B.; Rosa, B.B.; Santos, B.F.; Santos, E.F.; Schoeninger, K.; Smith, D.R.; Somavilla, A.; Tavares, M.T.; Zanella, F.C.V.; Zilch, K. 2018. Hymenoptera in Catálogo Taxonômico da Fauna do Brasil. PNUD. Avaiable at: http://fauna.jbrj.gov.br/fauna/faunadobrasil/96. Access in: 01/03/2018.

Onody, H.C.; Kawada, R. \& Brandão, C.R.F. 2014. Type specimens of Hymenoptera deposited in the Museu de Zoologia da Universidade de São Paulo, Brazil (excluding Aculeata). Papéis Avulsos de Zoologia, 54: 391-402. DOI

Packard, A.S. 1869. List of Hymenopterous and lepidopterous insects collected by the Smithsonian expedition to South America, under Prof. James Orton. Annual Report of the Trustees of the Peabody Academy Science, 1: 56-69.

Pilgrim, E.M.; von Dohlen, C.D. \& Pitts, J.P. 2008. Molecular phylogeneticsof Vespoidea indicate paraphyly of the superfamily and novel relationships of its componentfamilies and subfamilies. Zoologica Scripta, 37:539-560. DOI 
Prado, L.P. \& Brandão, C.R.F. 2013. A catalogue of Cephalotini ant type specimens (Hymenoptera: Formicidae: Myrmicinae) deposited in the Museu de Zoologia da Universidade de São Paulo, Brazil. Papéis Avulsos de Zoologia, 53: 285-293. DOI

Ramos, K.S.; Kawada, R. \& Brandão, C.R.F. 2015. Type specimens of bees (Hymenoptera, Apidae) deposited in the Museu de Zoologia da Universidade de São Paulo, Brazil. Papéis Avulsos de Zoologia, 53: 285-293. DOI

Raw, A. 2000. Mischocyttarus (Kappa) santacruzi, a new species of social wasp (Hymenoptera:Vespidae) from Eastern Brazilian wet forest. Revista Brasileira de Zoologia, 17: 941-943. DOI

Richards, 0.W. 1940. Preliminary diagnoses of some new species of Mischocyttarus Saussure (Hymenopt., Vespidae). Annals and Magazine of Natural History, 5: 176-183. D0l

Richards, 0.W. 1945. A revision of the genus Mischocyttarus de Saussare (Hymen., Vespidae). Transactions of the Royal Entomological Society London, 95: 295-462. D0I

Richards, 0.W. 1978. The social wasp of America, excluding the Vespinae. British Museum (Natural History), Publications, 785: 1-580.

Roig Alsina, A. 1981. Revision del género Entypus Dahlbom en la Argentina (Hymenoptera: Pompilidae). Revista de la Sociedad Entomologica de Argentina, 40: 311-336.

Santos, A.D.; Onody, H.C. \& Brandão, C.R.F. 2017. Type specimens of Chrysidoidea (Hymenoptera) deposited in the Museu de Zoologia da Universidade de São Paulo, Brazil. Papéis Avulsos de Zoologia, 57: 327-345. DOI

Schrottky, C. 1911. Neue südamerikanische Hymenoptera. Entomologische Rundschau, 28: 19-20.

Silveira, O.T. \& Carpenter, J.M. 1995. Protopolybia bituberculata, a new neotropical social wasp (Hymenoptera: Vespidae; Polistinae). Journal of the New York Entomological Society, 103: 48-54.

Suárez, F.J. 1960. Datos sobre mutílidos neotropicales I. Nuevas especies de Sphaerophthalminae (Hymenoptera). EOS, Revista Española de Entomologia, 36: 451-480.
Suárez, F.J. 1961. Datos sobre mutílidos neotropicales II. Dos nuevas especies de Argentina y Brasil (Hymenoptera). EOS, Revista Española de Entomologia, 37: 221-228.

Suárez, F.J. 1962. Datos sobre mutílidos neotropicales IV. Nuevas especies del género Lophomutilla Mickel (Hymenoptera: Mutillidae). Studia Entomologica, 5: 195-208.

Suárez, F.J. 1966. Datos sobre mutílidos neotropicales VI. Dos nuevos géneros de Pseudomethocini de Méjico y Brasil (Hymenoptera). EOS, Revista Española de Entomologia, 41: 471-482.

Ulysséa, M.A. \& Brandão, C.R.F. 2013. Catalogue of Dacetini and Solenopsidini ant type specimens (Hymenoptera, Formicidae, Myrmicinae) deposited in the Museu de Zoologia da Universidade de São Paulo, Brazil. Papéis Avulsos de Zoologia, 53: 187-209. D0I

Ulysséa, M.A.; Prado, L.P. \& Brandão, C.R.F. 2015. Type specimens of the traditional Myrmicinae (Hymenoptera: Formicidae) ant tribes deposited in the Museu de Zoologia da Universidade de São Paulo, Brazil: Adelomyrmecini, Basicerotini, Blepharidattini, Crematogastrini, Formicoxenini, Lenomyrmecini, Myrmicini, Phalocromyrmecini, Pheidolini, Stegomyrmecini, Stenammini and Tetramoriini. Papéis Avulsos de Zoologia, 55(12): 175-204.

Vardy, C.R. 2000. The New World tarântula-hawk wasp genus Pepsis Fabricius (Hymenoptera: Pompilidae). Part 1. Introduction and the P. rubra species group. Zoologische Verhandelingen Leiden, 332: 1-186.

Vardy, C.R. 2002. The New World tarantula-hawk wasp genus Pepsis Fabricius (Hymenoptera: Pompilidae). Part 2. The P. grossa - to P. deaurata groups. Zoologische Verhandelingen, Leiden, 337: 1-135.

Vardy, C.R. 2005. The New World tarantula-hawk wasp genus Pepsis Fabricius (Hymenoptera: Pompilidae). Part 3. The P. inclyta to auriguttata-groups. Zoologische Mededelingen, 79: 1-305.

Zikán, J.F. 1935. Die sozialen Wespen der Gattung Mischocyttarus Saussure, nebst Beschreibung von 27 neuen Arten (Hym., Vespidae). Arquivos do Instituto de Biologia Vegetal, 1: 143-243.

Zikán, J.F. 1949. 0 gênero Mischocyttarus Saussure (Hymenoptera, Vespidae), com a descrição de 82 espécies novas. Boletim do Parque Nacional do Itatiaia, 1: 1-251. 